\title{
Systematic review of infant and young child complementary feeding practices in South Asian families: the India perspective
}

\author{
Logan Manikam ${ }^{1, *}$, Ankita Prasad ${ }^{2}$, Abina Dharmaratnam ${ }^{3}$, Christy Moen ${ }^{4}$, \\ Alexandra Robinson ${ }^{5}$, Alexander Light ${ }^{2}$, Sonia Ahmed ${ }^{1}$, Raghu Lingam ${ }^{6}$ and \\ Monica Lakhanpaul ${ }^{1}$ \\ 'Population, Policy \& Practice, UCL Great Ormond Street Institute of Child Health, 30 Guilford Street, London WC1N \\ 1EH, UK: ${ }^{2}$ King's College London GKT School of Medical Education, London, UK: ${ }^{3}$ Leicester Medical School, \\ University of Leicester, Leicester, UK: ${ }^{4} \mathrm{St}$ George's University of London, London, UK: ${ }^{5} \mathrm{St}$ George's Hospital, London, \\ UK: 'Institute of Health \& Society, Newcastle University, Newcastle upon Tyne, UK
}

Submitted 8 November 2016: Final revision received 31 August 2017: Accepted 4 September 2017: First published online 23 November 2017

\begin{abstract}
Objective: Suboptimal nutrition among children remains a problem among South Asian (SA) families. Appropriate complementary feeding (CF) practices can greatly reduce this risk. Thus, we undertook a systematic review of studies assessing $\mathrm{CF}$ (timing, dietary diversity, meal frequency and influencing factors) in children aged $<2$ years in India.

Design: Searches between January 2000 and June 2016 in MEDLINE, EMBASE, Global Health, Web of Science, OVID Maternity \& Infant Care, CINAHL, Cochrane Library, BanglaJOL, POPLINE and WHO Global Health Library. Eligibility criteria: primary research on $\mathrm{CF}$ practices in SA children aged 0-2 years and/or their families. Search terms: 'children', 'feeding' and 'Asians' and derivatives. Two researchers undertook study selection, data extraction and quality appraisal (EPPICentre Weight of Evidence).

Results: From 45712 abstracts screened, sixty-four cross-sectional, seven cohort, one qualitative and one case-control studies were included. Despite adopting the WHO Infant and Young Child Feeding guidelines, suboptimal CF practices were found in all studies. In twenty-nine of fifty-nine studies, CF was introduced between 6 and 9 months, with eight studies finding minimum dietary diversity was achieved in $6-33 \%$, and ten of seventeen studies noting minimum meal frequency in only $25-50 \%$ of the study populations. Influencing factors included cultural influences, poor knowledge on appropriate CF practices and parental educational status.

Conclusions: This is the first systematic review to evaluate CF practices in SA in India. Campaigns to change health and nutrition behaviour and revision of nationwide child health nutrition programmes are needed to meet the substantial unmet needs of these children.
\end{abstract}

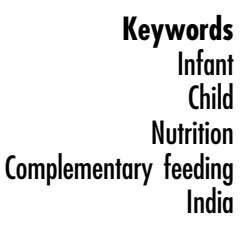

eywords

Infant
Child

Mutrition

India
Undernutrition including stunting and suboptimal breastfeeding accounts for $45 \%$ of all childhood deaths ${ }^{(1)}$. It is estimated that $30 \%$ of the world's stunted children live in Asia, with more than 60 million living in India; $31 \%$ of the developing world's total ${ }^{(1,2)}$. Inadequate complementary feeding (CF) has been linked to these outcomes.

The WHO defines CF as: "The process starting when breast milk alone is no longer sufficient to meet the nutritional requirements of infants, and therefore other foods and liquids are needed, along with breast milk ${ }^{(3)}$. CF therefore focuses on bridging the gradual transition between 6 and 24 months from exclusive breast-feeding to solid foods eaten by the whole family alongside breast-feeding.

Poor complementary feeding practices (CFP) have been linked to increased risks of respiratory and gastrointestinal infections, underweight and mortality ${ }^{(4-6)}$. $\mathrm{CF}$ is also important for reducing stunting, which is a current policy priority in India ${ }^{(7-9)}$. Despite this, in two published nonsystematic reviews on CFP in India, Ramji and Engle noted that CF was often started at inappropriate times ${ }^{(10,11)}$. 
There was also inappropriate quantities and diversity of complementary foods, with only $55 \%$ of South Asian (SA) infants consuming appropriate complementary foods by 6-8 months of age and growth retardation notable by 2 years of age ${ }^{(12,13)}$.

In policy, there has been recent increasing focus on CF. The 2010 WHO Infant and Young Child Feeding (IYCF) guidelines, an internationally ratified framework adopted in India, emphasize as a global public health recommendation that infants should be exclusively breast-fed for the first 6 months of life to achieve optimal growth, development and health ${ }^{(14)}$. Thereafter, infants should receive safe and nutritionally adequate complementary foods while breastfeeding continues for up to 2 years of age or beyond.

With no previously published systematic review identified, we aimed to assess the adequacy of CFP based on IYCF recommended criteria for minimum dietary diversity, meal frequency and timing of CF introduction. We also aimed to investigate barriers and promoters for appropriate CFP in SA children under 2 years old. By doing so, we hope to inform future work in developing and assessing the effectiveness of culturally appropriate interventions to improve CFP across these communities.

To limit the scope of our review, we focused on SA families residing in India, Pakistan, Bangladesh and high-income countries.

\section{Methods}

Due to the vast number of publications identified, the present review (PROSPERO registration number CRD42014014025) summarizes publications on CFP in SA families in India only, with concurrent reviews summarizing publications on CFP in SA families in high-income countries (L Manikam, R Lingam, I Lever et al., unpublished results), Pakistan $^{(15)}$ and Bangladesh ${ }^{(16)}$, respectively. High-income countries were included to investigate any differences in practice for SA who may have emigrated.

\section{Eligibility criteria}

Studies were included if they met the following criteria.

- Participants: children aged 0-2 years, parents, carers and/or their families.

- Outcomes: adequacy of CF (based on minimum dietary diversity and meal frequency), timing of introduction of $\mathrm{CF}$ and barriers/promoters to incorporating WHO recommended CFP.

- Language: studies published in English, or with translation available.

- Year: published from 2000 or later.

We excluded studies focusing solely on exclusive breast-feeding and interventional studies. Studies focusing on subgroups, such as children with co-morbidities, were considered eligible in principle.
In the IYCF indicators, introduction of CF is assessed as the proportion of infants aged 6-8 months who receive solid, semi-solid or soft foods. In contrast, minimum dietary diversity (MDD) is assessed by the proportion of children 6-23 months of age who receive foods from four or more food groups. The seven WHO IYCF recommended food groups are ${ }^{(14)}$ :

1. grains, roots and tubers;

2. legumes and nuts;

3. dairy products (e.g. milk, yoghurt, cheese);

4. flesh foods (e.g. meat, fish, poultry and liver/organ meats);

5. eggs;

6. vitamin A-rich fruits and vegetables; and

7. other fruits and vegetables.

While the consumption of Fe-rich or Fe-fortified foods is commonly assessed as a separate IYCF indicator, this was incorporated within dietary diversity for ease of interpretation in the current review.

Finally, minimum meal frequency (MMF) is assessed by the proportion of breast-fed and non-breast-fed children 6-23 months of age who receive solid, semi-solid or soft foods (also including milk feeds for non-breast-fed children) the minimum number of times or more per day: two times for 6-8 months, three times for 9-23 months and four times for 6-23 months (if not breast-fed).

Due to the nature of the topic, all study types (qualitative, quantitative or mixed) were included to ensure the diversity of evidence was captured and summarized, to be of relevance to both policy makers and health and social care professionals.

\section{Information sources}

A search strategy was devised to search the following databases: MEDLINE, BanglaJOL, EMBASE, CINAHL, Global Health, Web of Science, OVID Maternity \& Infant Care, The Cochrane Library, POPLINE and WHO Global Health Library. The WHO ICTRP (International Clinical Trials Registry Platform) was also searched. Searches were conducted in December 2014 and updated in June 2016.

Members of electronic networks on @jiscmail.ac.uk including minority-ethnic-health and networks (e.g. South Asian Health Foundation) developed from the Specialist Electronic Library for Ethnicity and Health were contacted to request any additional or unpublished material from members of the networks. We sought information specialist assistance to attempt to acquire unpublished material from each paper itself, and contacted study authors where possible. Bibliographies of included articles were also hand-searched for possible additional publications.

\section{Search strategy}

The search strategy included terms for 'feeding', 'South Asian' (including terms specifying all major subgroups) and 'children'. For example, the search strings used for MEDLINE were the following. 
Term 1: children $<2$ years

Infant OR Baby OR Babies OR Toddler OR Newborn OR Neonat* OR Child OR Preschool OR Nursery school OR Kid OR Pediatri* OR Minors OR Boy OR Girl

Term 2: feeding

Nutritional Physiological Phenomena OR Food OR Feeding behavior OR Feed OR Nutrition OR Wean OR fortif* OR Milk

Term 3: Asians

Ethni* OR India* OR Pakista* OR Banglades* OR Sri Lanka OR Islam OR Hinduism OR Muslim OR Indian subcontinent OR South Asia

\section{Study selection and data extraction}

In total, 45712 titles and abstracts were screened against inclusion criteria. Two reviewers assessed these papers independently, with conflicts resolved by discussion with the team. In view of the large number of articles deemed eligible for full-text review, articles published before the year 2000 were excluded. In total, 44852 titles and abstracts were excluded.

This left 860 potentially eligible full-text articles describing CFP in SA children, which were independently reviewed by two reviewers. One hundred and thirty-one full-text articles were ultimately extracted, of which seventy-three were relevant to India, seventeen were relevant to Pakistan, thirty-six were relevant to Bangladesh and ten were relevant to high-income countries.

Data were extracted by a single reviewer using a piloted modified worksheet including: country of study; study type; study year; study objectives; population studied, eligibility criteria and illness diagnosis; study design; ethical approval; sampling; data collection and analysis; feeding behaviours; adequacy of CFP; timing of initiation of CF; bias; value of the research; and weight of evidence. A second member of the research team checked each extraction, with further checking taking place as necessary.

\section{Result synthesis}

The eligible studies tended to address very broad research questions, were conducted using qualitative and/or quantitative and/or descriptive methods, and were not presented following standardized reporting guidelines (e.g. STROBE (Strengthening the Reporting of Observational Studies in Epidemiology) for observational studies or COREQ (Consolidated Criteria for Reporting Qualitative Research) for qualitative research). Meta-analyses were therefore not undertaken.

To standardize study classifications, the formal definitions below were utilized and applied ${ }^{(17,18)}$.
1. Intervention study: a study in which patients are assigned to a treatment or comparison group and followed prospectively.

2. Cohort study: an observational study in which a group of patients are followed over time. These may be prospective or retrospective.

3. Cross-sectional study: an observational study that examines the relationship between health-related characteristics and other variables of interest in a defined population at one particular time.

4. Case-control study: a study that compares patients who have a disease or outcome of interest (cases) with patients who do not have the disease or outcome (controls).

5. Qualitative: a study which aims to explore the experiences or opinions of families through interviews, focus groups, reflective field notes and other nonquantitative approaches.

6. Mixed methods: a study that combines both quantitative and qualitative methodology.

In view of the considerable heterogeneity among the studies identified in terms of methods, participants, interventions and outcomes, a narrative approach to synthesis was utilized using guidance developed from the University of York Centre for Reviews and Dissemination (CRD) and the Economic and Social Research Council (ESRC) ${ }^{(19-22)}$.

The evidence reviewed is presented as a narrative report, with results broadly categorized following IYFP indicators on: (i) adequacy of CFP, comprising dietary diversity, meal frequency, timing of introduction of CFP, consumption of Fe-rich foods and sources of advice for feeding; and (ii) barriers/promoters influencing CFP.

Barriers were defined as obstacles or impediments to achieving correct $\mathrm{CFP}^{(23)}$, while promoters were defined as supporters to achieving correct $\mathrm{CFP}^{(24)}$. These were sub-categorized into factors influencing at the family level (e.g. family members) and the organizational level (e.g. health-care providers, hospitals, political bodies).

\section{Quality assurance}

The CRD guidance emphasizes the importance of using a structured approach to quality assessment when assessing descriptive or qualitative studies for inclusion in reviews. However, it acknowledges the lack of consensus on the definition of poor quality with some arguing that using rigid quality criteria leads to the unnecessary exclusion of papers $^{(19)}$.

In our review, the EPPI-Centre Weight of Evidence Framework was used to allow objective judgements about each study's value in answering the review question. It examines three study aspects: quality of methodology, relevance of methodology and relevance of evidence to the review question, and categorizes them into 'low' (L), 'medium' (M) or 'high' $(\mathrm{H})^{(25)}$. An average of these weightings is taken to establish the study's overall weight of evidence (WOE), also rated as L, M or H. Two independent reviewers performed this evaluation, with 


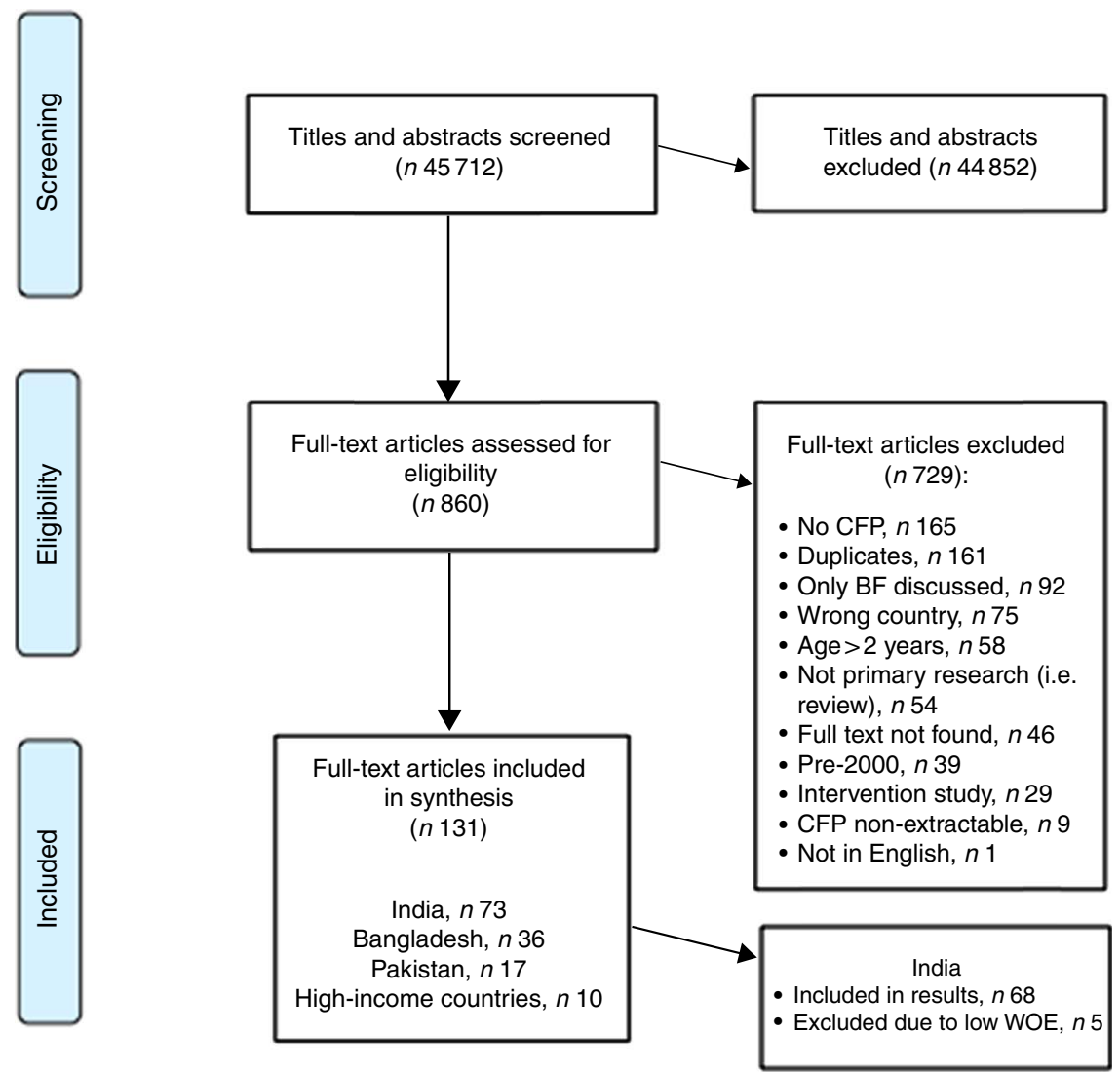

Fig. 1 Study selection process for the current systematic review (CFP, complementary feeding practices; BF, breast-feeding; WOE, weight of evidence)

additional arbitration by other team members where required. Studies with an overall $\mathrm{WOE}=\mathrm{L}$ are included in the table summarizing included studies but are not discussed further within the 'Results' or 'Discussion' section below.

\section{Results}

Of the 45712 studies identified, seventy-three studies focusing on CFP in SA families in India were ultimately included in the current systematic review. The study selection process is denoted in Fig. 1.

\section{Study and participant characteristics}

These seventy-three studies consisted of sixty-four crosssectional, seven cohort, one qualitative and one casecontrol. Sixty-eight studies met Weight of Evidence criteria and were included in the main results. Their participants included a total of 125326 children and 5705 mothers or caregivers when infants were not reported. Twenty-one studies reported details of the religion of participants, which was Hindu majority in nineteen samples and Muslim majority in two samples.

Table 1 summarizes all included studies. Figure 2 illustrates the study locations of sixty-three of these seventy-three included studies; the remaining nine do not detail precise study locations due to being described as 'national', 'various', 'urban' or 'rural' without specifics. Table 1 contains further details of study locations.

Table 2 presents the Weight of Evidence awarded to each of the studies. Thirteen studies had an overall WOE rating of $\mathrm{H}$, fifty-five studies an overall WOE rating of $\mathrm{M}$, and five studies had an overall WOE rating of $\mathrm{L}$.

The core narrative themes extracted from the papers are presented under the following headings: (i) adequacy of CFP and (ii) factors influencing CFP. The former is categorized further into dietary diversity, meal frequency, timing of introducing CF and advice providers.

\section{Adequacy of complementary feeding}

As per the WHO IYCF indicators, adequacy of CFP is assessed according to dietary diversity, meal frequency and timing of introducing CFP. These are detailed in the subsections below with a further subsection discussing advice providers.

\section{Dietary diversity}

Dietary diversity was measured in some form in fourteen studies. Rates of achieved MDD varied throughout studies but were generally low, with MDD achieved by between 6 and $33 \%$ of infants in eight studies that reported this 
Table 1 Summary of studies included in the current systematic review

\begin{tabular}{|c|c|c|c|c|c|}
\hline Study & Study type & Location & Population & $\begin{array}{l}\text { Sample } \\
\text { size }\end{array}$ & Adequacy of and factors influencing CFP \\
\hline $\begin{array}{l}\text { Aggarwal et al. } \\
(2008)^{(57)}\end{array}$ & Cross-sectional & Delhi, India & $\begin{array}{l}\text { Mothers of infants } \\
6 \text { months to } 2 \text { years } \\
\text { old attending } \\
\text { outpatient paediatric } \\
\text { hospital }\end{array}$ & 200 & $\begin{array}{l}\text { Frequency: Frequency of complementary feeds was less than recommended in about } 75 \% \text { of children } \\
\text { Factors: Maternal and paternal education, lack of knowledge regarding CF, child vomiting, advice from } \\
\text { family elders } \\
\text { Timing: } 34 \% \text { started up to } 1 \text { year }\end{array}$ \\
\hline $\begin{array}{l}\text { Aguayo et al. } \\
(2016)^{(50)}\end{array}$ & Cross-sectional & Maharashtra, India & $\begin{array}{l}\text { Children under } \\
23 \text { months old }\end{array}$ & 2561 & $\begin{array}{l}\text { Diversity: } 6 \% \text { of } 6-23 \text {-month-olds were fed } 4+\text { food groups } \\
\text { Frequency: } 77 \% \text { of } 6-23 \text {-month-olds met MMF. When sick, many children (up to } 75 \% \text { ) see their complementary } \\
\text { foods restricted in frequency } \\
\text { Factors: Poor sanitation, mother's nutrition status, poverty } \\
\text { Timing: } 59 \% \text { of } 6-8 \text {-month-olds had CFP introduced }\end{array}$ \\
\hline $\begin{array}{l}\text { Aruldas et al. } \\
(2010)^{(26)}\end{array}$ & Cross-sectional & Rural India & $\begin{array}{l}\text { Children aged } \\
0-23 \text { months }\end{array}$ & 4472 & $\begin{array}{l}\text { Diversity: } 30 \% \text { were fed at least three types of food as recommended } \\
\text { Frequency: } 63 \% \text { of children aged } 6-23 \text { months were given the minimum recommended number of } \\
\text { feeds in a day } \\
\text { Factors: } 56 \% \text { of mothers commenced CF before } 6 \text { months of age because they felt that their breast milk } \\
\text { was not sufficient for the child. Other factors include high standard of living, education, media exposure } \\
\text { and ANC check-ups } \\
\text { Timing: } 46 \% \text { at } 7-9 \text { months }\end{array}$ \\
\hline $\begin{array}{l}\text { Bagul and Supare } \\
(2012)^{(66)}\end{array}$ & Cross-sectional & $\begin{array}{l}\text { Urban slum of Nagpur, } \\
\text { Maharashtra, India }\end{array}$ & $\begin{array}{l}\text { Children under } 1 \text { year } \\
\text { old }\end{array}$ & 384 & $\begin{array}{l}\text { Factors: Literacy } \\
\text { Timing: } 51 \% \text { under } 6 \text { months }\end{array}$ \\
\hline $\begin{array}{l}\text { Bahuguna et al. } \\
(2013)^{(98)}\end{array}$ & Case-control & Uttar Pradesh, India & $\begin{array}{l}\text { Children aged 1-18 } \\
\text { years }\end{array}$ & 800 & Diversity: Milk, sweets, fruits were eaten, but this was not broken down by age \\
\hline $\begin{array}{l}\text { Bentley et al. } \\
\quad(2015)^{(27)}\end{array}$ & Cross-sectional & $\begin{array}{l}\text { Informal settlements, } \\
\text { Mumbai, India }\end{array}$ & Children under 5 years & 7450 & $\begin{array}{l}\text { Diversity: Dietary diversity was limited ( } 13 \%) \\
\text { Frequency: MMF was met by less than half of infants } \\
\text { Timing: } 41 \% \text { commenced at } 6-8 \text { months }\end{array}$ \\
\hline $\begin{array}{l}\text { Bhandari et al. } \\
(2002)^{(49)}\end{array}$ & Cross-sectional & Delhi, India & $\begin{array}{l}\text { Children aged } \\
12-23 \text { months }\end{array}$ & 395 & $\begin{array}{l}\text { Factors: Education } \\
\text { Timing: Animal milk given at mean age of } 3 \text { months }\end{array}$ \\
\hline $\begin{array}{l}\text { Bhanderi and } \\
\text { Choudhary } \\
(2011)^{(64)}\end{array}$ & Cross-sectional & $\begin{array}{l}\text { Petlad town, a semi- } \\
\text { urban area of Anand } \\
\text { district, Gujarat, } \\
\text { India }\end{array}$ & $\begin{array}{l}\text { Children under } 5 \text { years } \\
\text { old }\end{array}$ & 300 & $\begin{array}{l}\text { Diversity: Rice, daal, curd, butter milk, ice creams, fruits } \\
\text { Factors: Educated mothers were more receptive to the message of proper weaning passed to them during } \\
\text { antenatal visits. Other factors include maternal education, place of delivery and sociocultural beliefs } \\
\text { Timing: } 52 \% \text { at } 4-6 \text { months }\end{array}$ \\
\hline $\begin{array}{l}\text { Caleyachetty et al. } \\
(2013)^{(63)}\end{array}$ & Cohort & $\begin{array}{l}\text { Mysore city or } \\
\text { surrounding rural } \\
\text { villages, India }\end{array}$ & $\begin{array}{l}\text { Mothers attending the } \\
\text { ANC of the } \\
\text { Holdsworth Memorial } \\
\text { Hospital }\end{array}$ & 830 & $\begin{array}{l}\text { Factors: Hindu mothers commenced CF later } v \text {. Islam or 'other' religions. Other factors include higher education } \\
\text { and lower socio-economic status } \\
\text { Timing: } 38 \% \text { at } 4 \text { months, } 27 \% \text { at } 5 \text { months }\end{array}$ \\
\hline $\begin{array}{l}\text { Chandwani et al. } \\
(2015)^{(28)}\end{array}$ & Cross-sectional & $\begin{array}{l}\text { Rural Health Training } \\
\text { Centre at Dabhoda, } \\
\text { Gujarat, India }\end{array}$ & $\begin{array}{l}\text { Children 0-24 months } \\
\text { old }\end{array}$ & 300 & $\begin{array}{l}\text { Diversity: } 28.3 \% \text { were given food from four or more groups } \\
\text { Frequency: MMF was adequate in } 95.6 \% \\
\text { Timing: } 60 \% \text { at } 6 \text { months }\end{array}$ \\
\hline $\begin{array}{l}\text { Chhabra and } \\
\text { Gupta }(2015)^{(87)}\end{array}$ & Cross-sectional & $\begin{array}{l}\text { Urbanized village of } \\
\text { East Delhi, India }\end{array}$ & $\begin{array}{l}\text { Children aged } \\
0-23 \text { months }\end{array}$ & 194 & $\begin{array}{l}\text { Factors: Wealth and gender of infant, born in government institution } \\
\text { Timing: } 6-9 \text { months for } 54 \%\end{array}$ \\
\hline $\begin{array}{l}\text { Chhabra et al. } \\
(2010)^{(53)}\end{array}$ & Cross-sectional & Ludhiana, India & $\begin{array}{l}\text { Children under } \\
12 \text { months old }\end{array}$ & 204 & $\begin{array}{l}\text { Diversity: Dal soup, juice, tea, kheer, banana, khichri all used } \\
\text { Factors: Mothers believing size was a more important indicator than age } \\
\text { Timing: Less than } 3 \text { months }\end{array}$ \\
\hline $\begin{array}{l}\text { Collison et al. } \\
(2015)^{(55)}\end{array}$ & Cross-sectional & $\begin{array}{l}\text { One urban and one } \\
\text { rural community in } \\
\text { Samastipur district, } \\
\text { Bihar, India }\end{array}$ & $\begin{array}{l}\text { Children preterm to } \\
18 \text { months old }\end{array}$ & 60 & $\begin{array}{l}\text { Diversity: Eggs, meat, fish, fruits, vegetables were used } \\
\text { Frequency: Mothers 'generally' feed infants two or three times each day } \\
\text { Factors: Urban and rural status affected feeding practices }\end{array}$ \\
\hline $\begin{array}{l}\text { D'Alimonte et al. } \\
(2016)^{(35)}\end{array}$ & Cross-sectional & $\begin{array}{l}\text { Slum, Dharavi, } \\
\text { Mumbai, India }\end{array}$ & $\begin{array}{l}\text { Mothers of children } \\
\text { under } 3 \text { years old }\end{array}$ & 22 & $\begin{array}{l}\text { Diversity: Listed } 7 \text { IYCF groups. Gave MDD details stratified by positive deviance. MDD achieved by majority at } \\
6 \text { months } \\
\text { Factors: Advice sources included female elders, relatives, community health workers, the media } \\
\text { Timing: Positive deviants mostly around } 6 \text { months }\end{array}$ \\
\hline $\begin{array}{l}\text { Dahiya and Sehgal } \\
(2002)^{(86)}\end{array}$ & Cross-sectional & Haryana, India & $\begin{array}{l}\text { Mothers of children } \\
\text { aged 6-18 months }\end{array}$ & 100 & $\begin{array}{l}\text { Diversity: Khichri, dalia, rice, kheer, fruit and vegetables were used } \\
\text { Factors: Only a few mothers belonging to high socio-economic status give ready-made foods to their infants } \\
\text { Timing: Working mothers started before } 6 \text { months, non-working mothers after }\end{array}$ \\
\hline
\end{tabular}


Table 1 Continued

\begin{tabular}{|c|c|c|c|c|c|}
\hline Study & Study type & Location & Population & $\begin{array}{l}\text { Sample } \\
\text { size }\end{array}$ & Adequacy of and factors influencing CFP \\
\hline $\begin{array}{l}\text { Dakshayani and } \\
\text { Gangadhar } \\
(2008)^{(99)}\end{array}$ & Cross-sectional & Karnataka, India & $\begin{array}{l}\text { Mothers of children } \\
\text { aged 0-60 months }\end{array}$ & 125 & $\begin{array}{l}\text { Factors: The practice of giving the infants some special type of feeds before initiating breast milk is widespread in } \\
\text { tribal areas } \\
\text { Timing: } 48 \% \text { at } 6-9 \text { months }\end{array}$ \\
\hline $\begin{array}{l}\text { Damayanthi et al. } \\
(2013)^{(72)}\end{array}$ & Cross-sectional & Bangalore, India & $\begin{array}{l}\text { Mothers of children } \\
\text { under } 24 \text { months }\end{array}$ & 300 & $\begin{array}{l}\text { Factors: Earlier initiation when literate } \\
\text { Timing: } 70 \% \text { at } 3-6 \text { months }\end{array}$ \\
\hline de Onis $(2006)^{(34)}$ & Cross-sectional & India & $\begin{array}{l}\text { Children aged } \\
0-24 \text { months }\end{array}$ & 8440 & $\begin{array}{l}\text { Diversity: MDD at different ages among compliant Indian children was: } 2.8 \text { foods at } 6 \text { months, } 4.1 \text { at } 9 \text { months, } \\
4.6 \text { at } 12 \text { months, } 4.9 \text { at } 18 \text { months, } 5.1 \text { at } 24 \text { months } \\
\text { Frequency: Frequency was around a mean of } 2 \text { non-milk meals per day among compliant children in India at } \\
5 \text { months, rising to } 2.8 \text { at } 6 \text { months, } 4 \text { at } 8 \text { months, } 4.9 \text { at } 12 \text { months, } 5.4 \text { at } 18 \text { months and } 5.5 \text { at } 24 \text { months } \\
\text { Timing: Mean timing was } 5 \text { months }\end{array}$ \\
\hline $\begin{array}{l}\text { Dibley et al: } \\
\quad(2010)^{(91)}\end{array}$ & Cross-sectional & $\begin{array}{l}29 \text { unnamed states } \\
\text { in India }\end{array}$ & $\begin{array}{l}\text { Last-born children aged } \\
0 \text { to } 23 \text { months living } \\
\text { with the respondent }\end{array}$ & 20108 & $\begin{array}{l}\text { Factors: Antenatal visits, media, living North, East or South } \\
\text { Timing: } 57 \% \text { timely CF rate }\end{array}$ \\
\hline $\begin{array}{l}\text { Fall et al. } \\
\qquad(2010)^{(100)}\end{array}$ & Cohort & $\begin{array}{l}\text { Brazil/Guatemala/ } \\
\text { India/Philippines/ } \\
\text { South Africa; New } \\
\text { Delhi in India }\end{array}$ & $\begin{array}{l}\text { Babies born to women } \\
\text { in an area of Delhi }\end{array}$ & 1526 & Timing: $42 \%$ at $9-12$ months \\
\hline $\begin{array}{l}\text { Farzani and Devi } \\
(2011)^{(68)}\end{array}$ & Cross-sectional & Parbhani district, India & $\begin{array}{l}\text { Mothers of children } \\
\text { aged 3-18 months }\end{array}$ & 130 & $\begin{array}{l}\text { Diversity: Cow's milk, honey, castor oil, dhal, fruit and vegetables } \\
\text { Factors: Literacy and maternal education } \\
\text { Timing: } 52 \% \text { at } 3-6 \text { months }\end{array}$ \\
\hline $\begin{array}{l}\text { Fazilli et al. } \\
\qquad(2011)^{(40)}\end{array}$ & Cross-sectional & Kashmir, India & $\begin{array}{l}\text { Multiparous women } \\
\text { attending the ANC of } \\
\text { the maternity hospital } \\
\text { of Sheri Kashmir } \\
\text { Institute of Medical } \\
\text { Sciences }\end{array}$ & 585 & $\begin{array}{l}\text { Diversity: Cereals, fruits, banana were used } \\
\text { Factors: Many harmful infant feeding practices still hold ground in the community, having their roots in cultural } \\
\text { influences and lack of knowledge regarding CFP timing } \\
\text { Timing: } 38 \% \text { at } 6-12 \text { months, } 32 \% \text { at } 6 \text { months }\end{array}$ \\
\hline $\begin{array}{l}\text { Garg and Chadha } \\
(2009)^{(36)}\end{array}$ & Cross-sectional & $\begin{array}{l}\text { Six villages of } \\
\text { Ghaziabad district, } \\
\text { Uttar Pradesh, India }\end{array}$ & $\begin{array}{l}\text { Mothers of children } \\
\text { aged 6-12 months }\end{array}$ & 151 & $\begin{array}{l}\text { Diversity: } 31 \text { and } 18 \% \text { of the mothers of the } 6-8 \text { - and } 9-12 \text {-month-old infants reported feeding } \geq 3 \text { and } \geq 4 \text { food } \\
\text { groups, respectively, to their infants in the preceding } 24 \mathrm{~h} \text {. Mothers used starchy staples, legumes, milk eggs, } \\
\text { others } \\
\text { Frequency: } 60 \% \text { of the mothers fed their infants the recommended number of meals in the previous } 24 \mathrm{~h} \\
\text { Factors: Wealth led to better practices. More children led to worse parity. Other factors include socio-economic } \\
\text { factors and maternal education }\end{array}$ \\
\hline $\begin{array}{l}\text { Goswami et al. } \\
\qquad(2012)^{(76)}\end{array}$ & Cross-sectional & $\begin{array}{l}5 \text { villages of the } \\
\text { Nuapadhi Gram } \\
\text { Panchayat, Remuna } \\
\text { block of Balasore } \\
\text { district of Orissa, } \\
\text { India }\end{array}$ & $\begin{array}{l}\text { Mothers of children } \\
\text { aged } 0-60 \text { months }\end{array}$ & 121 & Timing: $61 \%$ initiate at $6-9$ months \\
\hline $\begin{array}{l}\text { Holambe and } \\
\text { Thakur } \\
(2014)^{(101)}\end{array}$ & Cross-sectional & Maharashtra, India & $\begin{array}{l}\text { Mothers attending } \\
\text { immunization OPD } \\
\text { with their infants (age } \\
\text { of baby }<12 \text { months) }\end{array}$ & 197 & $\begin{array}{l}\text { Factors: Maternal age, education, siblings } \\
\text { Timing: } 46 \% \text { started CF at appropriate age }\end{array}$ \\
\hline $\begin{array}{l}\text { Jayant et al. } \\
\quad(2010)^{(67)}\end{array}$ & Cross-sectional & Loni, India & $\begin{array}{l}\text { Mothers of children } \\
\text { aged } 0-5 \text { years } \\
\text { attending } \\
\text { immunization clinic } \\
\text { and paediatric OPD }\end{array}$ & 300 & $\begin{array}{l}\text { Diversity: Milk and water mentioned } \\
\text { Factors: Knowledge and support, education on CFP timing } \\
\text { Timing: } 42 \% \text { at } 6-8 \text { months }\end{array}$ \\
\hline Jindal $(2009)^{(102)}$ & Cross-sectional & Mangalore, India & $\begin{array}{l}\text { Mothers of infants aged } \\
6-12 \text { months during } \\
\text { their visits at the OPD } \\
\text { of two hospitals }\end{array}$ & 104 & $\begin{array}{l}\text { Diversity: Fruit juice and ragi porridge } \\
\text { Factors: Early, inadequate expressing. Late }=\text { child refusing to eat } \\
\text { Timing: Majority weaned before } 6 \text { months with fruit juice }\end{array}$ \\
\hline $\begin{array}{l}\text { Kapur et al. } \\
(2005)^{(43)}\end{array}$ & Cross-sectional & $\begin{array}{l}\text { Urban slum, Delhi, } \\
\text { India }\end{array}$ & $\begin{array}{l}\text { Children 9-36 months of } \\
\text { age, in an urban slum } \\
\text { Integrated Child } \\
\text { Development } \\
\text { Services project }\end{array}$ & 545 & $\begin{array}{l}\text { Diversity: Cereals, pulses, flesh foods, milk, vegetables, others mentioned } \\
\text { Factors: Gender of child }\end{array}$ \\
\hline
\end{tabular}




\begin{tabular}{|c|c|c|c|c|c|}
\hline Study & Study type & Location & Population & $\begin{array}{l}\text { Sample } \\
\text { size }\end{array}$ & Adequacy of and factors influencing CFP \\
\hline $\begin{array}{l}\text { Katara et al. } \\
(2013)^{(37)}\end{array}$ & Cross-sectional & Urban slums, India & $\begin{array}{l}\text { Children aged } \\
6-24 \text { months }\end{array}$ & 561 & $\begin{array}{l}\text { Diversity: } 64.7 \% \text { were given an appropriate number of food groups. Cereals, pulses, fruits/vegetables, milk } \\
\text { mentioned } \\
\text { Frequency: } 25 \% \text { of children were receiving adequate frequency of CF and its association with gender was significant } \\
\text { Factors: Parents thinking their child is too old for breast milk after } 6 \text { months, high birth order, gender, maternal } \\
\text { literacy, young mothers } \\
\text { Timing: } 60.5 \% \text { after } 6 \text { months }\end{array}$ \\
\hline $\begin{array}{l}\text { Khan et al. } \\
(2012)^{(33)}\end{array}$ & Cross-sectional & $\begin{array}{l}\text { Urban health centres of } \\
\text { the Department of } \\
\text { Community } \\
\text { Medicine of UCMS, } \\
\text { East Delhi, India }\end{array}$ & $\begin{array}{l}\text { Children less } 24 \text { months } \\
\text { old who were } \\
\text { attending an } \\
\text { immunization clinic }\end{array}$ & 374 & $\begin{array}{l}\text { Diversity: MDD was observed in } 32.6 \% \text { of the children aged } 6-23 \text { months } \\
\text { Frequency: MMF was observed in } 48.6 \% \text { of the children }\end{array}$ \\
\hline $\begin{array}{l}\text { Kumar et al. } \\
(2006)^{(103)}\end{array}$ & Cross-sectional & $\begin{array}{l}4 \text { anganwari areas of } \\
\text { urban Allahbad, } \\
\text { Uttar Pradesh, India }\end{array}$ & $\begin{array}{l}\text { Children aged under } \\
60 \text { months }\end{array}$ & 217 & $\begin{array}{l}\text { Diversity: WHO recommended food was used } \\
\text { Factors: Knowledge } \\
\text { Timing: } 48 \% \text { practised CF during } 6-9 \text { months }\end{array}$ \\
\hline $\begin{array}{l}\text { Kumar et al. } \\
(2013)^{(46)}\end{array}$ & Cross-sectional & Rural Tumkur, India & Lactating mothers & 110 & $\begin{array}{l}\text { Diversity: Ragi sari, biscuits, Cerelac, cow's milk, Farex, goat's milk } \\
\text { Frequency: } 46 \% \text { given weaning food twice daily and } 31 \% \text { once daily } \\
\text { Factors: Rural areas }\end{array}$ \\
\hline $\begin{array}{l}\text { Kuriakose } \\
\qquad(2010)^{(59)}\end{array}$ & Cross-sectional & Karnataka, India & $\begin{array}{l}\text { Randomly selected } \\
\text { infants from } \\
\text { Karnataka }\end{array}$ & 112 & $\begin{array}{l}\text { Frequency: } 47 \% \text { given } C F \text { four times daily } \\
\text { Factors: Number of children inversely proportional to quality of CFP, educational status of mother improved } \\
\text { Timing: } 31 \% \text { started CF at } 4 \text { months }\end{array}$ \\
\hline $\begin{array}{l}\text { Lingam et al. } \\
(2014)^{(52)}\end{array}$ & Qualitative & Rural Rajasthan, India & $\begin{array}{l}\text { Children aged } \\
0-24 \text { months }\end{array}$ & 87 & $\begin{array}{l}\text { Diversity: Cerelac, porridge, biscuits, roti, milk, rice, almonds, lentils were used } \\
\text { Factors: Lack of advice, poor families } \\
\text { Timing: Often a delay until 7-12 months of age }\end{array}$ \\
\hline $\begin{array}{l}\text { Lohia and Udipi } \\
(2014)^{(39)}\end{array}$ & Cohort & $\begin{array}{l}6 \text { urban slums in } 3 \\
\text { western suburbs, } \\
\text { Mumbai, India }\end{array}$ & $\begin{array}{l}\text { Children aged } \\
6-24 \text { months }\end{array}$ & 446 & $\begin{array}{l}\text { Diversity: Scores provided by age, IYCF groups measured } \\
\text { Frequency: Over half of males }(54.8 \%)<12 \text { months of age had a higher feeding frequency score } v \text {. one-third of } \\
\text { females }(32.7 \%) \text { at the same age } \\
\text { Factors: Maternal education, male child, age, BMI of mother }\end{array}$ \\
\hline Malhotra $(2013)^{(38)}$ & Cross-sectional & India - national & $\begin{array}{l}\text { Children aged } \\
6-18 \text { months }\end{array}$ & 9241 & $\begin{array}{l}\text { Diversity: MDD at } 6-8 \text { months } 3 \% \text {, at } 9-11 \text { months } 9 \% \text {, at } 12-18 \text { months } 17 \% \\
\text { Frequency: MMF at } 6-8 \text { months } 25 \% \text {, at } 9-11 \text { months } 39 \% \text {, at } 12-18 \text { months } 54 \% \\
\text { Factors: Illness, siblings, health-care professionals' advice, media, mother working from home } \\
\text { Timing: } 63 \% \text { had commenced weaning by } 6-8 \text { months }\end{array}$ \\
\hline $\begin{array}{l}\text { Mayuri et al. } \\
\quad(2012)^{(79)}\end{array}$ & Cross-sectional & India-four zones & $\begin{array}{l}\text { Infants from eight } \\
\text { centres from different } \\
\text { states across four } \\
\text { zones (North, East, } \\
\text { South and West) in } \\
\text { India }\end{array}$ & 800 & $\begin{array}{l}\text { Diversity: Milk, biscuits, fennel seeds, cardamom, cereals } \\
\text { Factors: Perception of insufficient milk, being tired after labour, convenience and as per elders' advice }\end{array}$ \\
\hline $\begin{array}{l}\text { Menon et al. } \\
(2015)^{(29)}\end{array}$ & Cross-sectional & India-national & $\begin{array}{l}\text { Children } 0-24 \text { months } \\
\text { old }\end{array}$ & 18463 & $\begin{array}{l}\text { Diversity: Grains, legumes, eggs, meat, fish. } 16 \% \text { of } 6-23 \text {-month-olds met MDD } \\
\text { Frequency: } 45 \% \text { met MMF } \\
\text { Factors: Education, delaying age of marriage, poverty, illiteracy } \\
\text { Timing: } 58 \% \text { used CF before } 6 \text { months }\end{array}$ \\
\hline $\begin{array}{l}\text { Meshram et al. } \\
(2012)^{(42)}\end{array}$ & Cross-sectional & Andhra Pradesh, India & $\begin{array}{l}\text { Child-mother pairs were } \\
\text { included using } \\
\text { systematic random } \\
\text { sampling }\end{array}$ & 805 & $\begin{array}{l}\text { Diversity: Cow/buffalo milk, home-made semi-solid foods e.g. cereals } \\
\text { Frequency: } 95 \% \text { received CF three times daily } \\
\text { Factors: Timely initiation was more likely among certain castes and tribes } \\
\text { Timing: Classified as } 6-9 \text { months }\end{array}$ \\
\hline $\begin{array}{l}\text { Meshram et al. } \\
\qquad(2013)^{(69)}\end{array}$ & Cross-sectional & $\begin{array}{l}\text { Rural Madhya } \\
\text { Pradesh, India }\end{array}$ & $\begin{array}{l}\text { Children under } 1 \text { year } \\
\text { old }\end{array}$ & 5457 & $\begin{array}{l}\text { Factors: Caste, literacy, wealth } \\
\text { Timing: } 50 \% \text { at } 6-8 \text { months }\end{array}$ \\
\hline $\begin{array}{l}\text { Mukhopadhyay } \\
\text { et al. }(2013)^{(30)}\end{array}$ & Cross-sectional & $\begin{array}{l}2 \text { slums, West Bengal, } \\
\text { India }\end{array}$ & $\begin{array}{l}\text { Children aged } \\
0-23 \text { months from } \\
2 \text { slums via two-stage } \\
\text { random sampling } \\
\text { technique }\end{array}$ & 245 & $\begin{array}{l}\text { Diversity: MDD was } 24.4 \% \\
\text { Frequency: Age-appropriate MMF was found in } 67.0 \% \text { children } \\
\text { Timing: } 12 \% \text { were before } 6 \text { months }\end{array}$ \\
\hline
\end{tabular}




\begin{tabular}{|c|c|c|c|c|c|}
\hline Study & Study type & Location & Population & $\begin{array}{l}\text { Sample } \\
\text { size }\end{array}$ & Adequacy of and factors influencing CFP \\
\hline $\begin{array}{l}\text { Narayanappa et al. } \\
(2015)^{(54)}\end{array}$ & Cross-sectional & Rural Karnataka, India & $\begin{array}{l}\text { Children 9-10 months } \\
\text { old }\end{array}$ & 957 & $\begin{array}{l}\text { Diversity: Janam ghutti, peanuts, Cerelac, animal milk, biscuits, rice with lentils, others } \\
\text { Factors: Education on how to maintain sufficient breast milk production and appropriate age for weaning } \\
\text { Timing: } 66 \% \text { before } 6 \text { months }\end{array}$ \\
\hline $\begin{array}{l}\text { Neog and Baruah } \\
\qquad(2012)^{(41)}\end{array}$ & Cross-sectional & $\begin{array}{l}\text { Jorhat district, Assam, } \\
\text { India }\end{array}$ & $\begin{array}{l}\text { Children aged } \\
1-12 \text { months }\end{array}$ & 120 & $\begin{array}{l}\text { Diversity: Milk, dal, rice, Cerelac, banana, luthri, khichri, cooked rice } \\
\text { Factors: Community norms, education } \\
\text { Timing: } 26 \% \text { given CF early }\end{array}$ \\
\hline $\begin{array}{l}\text { Padhy and } \\
\text { Choudhury } \\
(2004)^{(78)}\end{array}$ & Cross-sectional & Orissa, India & $\begin{array}{l}\text { Mothers of children } \\
\text { under } 12 \text { months }\end{array}$ & 131 & $\begin{array}{l}\text { Factors: Poverty, tradition, knowledge } \\
\text { Timing: } 63 \% \text { between } 3 \text { and } 6 \text { months }\end{array}$ \\
\hline $\begin{array}{l}\text { Padmadas et al. } \\
\quad(2002)^{(62)}\end{array}$ & Cross-sectional & 6 regions of India & Children $24-47$ months & 6285 & $\begin{array}{l}\text { Factors: Later start in Central and East (except West Bengal) India, maternal education } \\
\text { Timing: } 53.5 \% \text { weaned at }<6 \text { months }\end{array}$ \\
\hline $\begin{array}{l}\text { Pasricha et al. } \\
\quad(2011)^{(44)}\end{array}$ & Cross-sectional & $\begin{array}{l}2 \text { rural districts of } \\
\text { Karnataka, India }\end{array}$ & $\begin{array}{l}\text { Children aged } \\
12-23 \text { months }\end{array}$ & 396 & $\begin{array}{l}\text { Diversity: Idli and dosa with rice and lentils, sambar, rice, ragi } \\
\text { Factors: Poverty and food insecurity increase breast-feeding }\end{array}$ \\
\hline $\begin{array}{l}\text { Passi and Shad } \\
(2004)^{(104)}\end{array}$ & Cross-sectional & $\begin{array}{l}\text { Tea garden in Assam, } \\
\text { India }\end{array}$ & $\begin{array}{l}\text { Children aged } \\
0-12 \text { months }\end{array}$ & 110 & $\begin{array}{l}\text { Factors: Poverty and illiteracy } \\
\text { Timing: Commenced by } 9-10 \text { months for } 56 \%\end{array}$ \\
\hline $\begin{array}{l}\text { Patel et al. } \\
(2012)^{(31)}\end{array}$ & Cross-sectional & India - national & $\begin{array}{l}\text { Last-born children aged } \\
6-23 \text { months }\end{array}$ & 15028 & $\begin{array}{l}\text { Diversity: Among children aged } 6-23 \text { months, MDD rate was } 15 \cdot 2 \% \text {. Foods included potatoes, bread, noodles, } \\
\text { milk, flesh foods, chicken, grains, roots, tubers } \\
\text { Frequency: When sick, many children (up to } 75 \% \text { ) see their complementary foods restricted in frequency. } 41.5 \% \\
\text { of } 6-23 \text {-month-olds met MMF } \\
\text { Factors: North and West India had higher odds of suffering from poor CFP; education, antenatal care } \\
\text { Timing: } 55 \% \text { aged } 6-8 \text { months were introduced to solid foods }\end{array}$ \\
\hline $\begin{array}{l}\text { Pathi et al. } \\
\quad(2003)^{(73)}\end{array}$ & Cross-sectional & $\begin{array}{l}\text { Rural block of Orissa, } \\
\text { India }\end{array}$ & $\begin{array}{l}\text { Children aged under } \\
1 \text { year }\end{array}$ & 383 & $\begin{array}{l}\text { Factors: Lack of awareness regarding proper weaning practices, education } \\
\text { Timing: } 36 \% \text { at } 8-12 \text { months }\end{array}$ \\
\hline $\begin{array}{l}\text { Rangaswamy et al. } \\
(2013)^{(45)}\end{array}$ & Cross-sectional & $\begin{array}{l}\text { Nagavalli in Tumkur, } \\
\text { India }\end{array}$ & $\begin{array}{l}\text { Children under } 1 \text { year of } \\
\text { age }\end{array}$ & 110 & $\begin{array}{l}\text { Diversity: Biscuits, Cerelac, cow's milk, Farex, ragi porridge, rice, dhal, others } \\
\text { Factors: Elderly family members were prominent influencers in decision when to add CF } \\
\text { Timing: } 46 \% \text { at } 4-6 \text { months }\end{array}$ \\
\hline Rao et al. $(2011)^{(80)}$ & Cross-sectional & Mangalore, India & $\begin{array}{l}\text { Mothers of children } \\
\text { aged 6-24 months }\end{array}$ & 200 & $\begin{array}{l}\text { Diversity: Ragi, wheat and rice } \\
\text { Factors: Number of children inversely proportional to quality of CFP. Education, birth location } \\
\text { Timing: } 78 \% \text { had started CF at recommended time }\end{array}$ \\
\hline $\begin{array}{l}\text { Rasania and } \\
\text { Sachdev } \\
(2001)^{(58)}\end{array}$ & Cross-sectional & Mehrauli, Delhi, India & $\begin{array}{l}\text { Children aged under } \\
5 \text { years old }\end{array}$ & 354 & $\begin{array}{l}\text { Diversity: Top milk was given } \\
\text { Frequency: } 46 \% \text { received } 5-8 \text { meals/d } \\
\text { Factors: Education } \\
\text { Timing: Weaning times ranged from before } 4 \text { months to after } 12 \text { months }\end{array}$ \\
\hline Roy et al. $(2009)^{(56)}$ & Cross-sectional & $\begin{array}{l}\text { Urban health centre, } \\
\text { Chetla, Kolkata, } \\
\text { India }\end{array}$ & $\begin{array}{l}\text { Children aged } \\
6-24 \text { months }\end{array}$ & 121 & $\begin{array}{l}\text { Diversity: Rice, dal, mashed potato, suji } \\
\text { Factors: Health facility, guardian and peer groups } \\
\text { Timing: } 71.7 \% \text { at } 6 \text { months }\end{array}$ \\
\hline $\begin{array}{l}\text { Samuel et al. } \\
(2012)^{(51)}\end{array}$ & Cohort & Bangalore, India & $\begin{array}{l}\text { Mothers of children } \\
\text { aged } 0-6 \text { months }\end{array}$ & 50 & $\begin{array}{l}\text { Diversity: Commercial cereal and milk, biscuits, mixed-grain porridges, rice and lentil cakes, others } \\
\text { Factors: Reasons for the early introduction of CF included a crying infant, employment, elders } \\
\text { Timing: } 64 \% \text { by } 6 \text { months }\end{array}$ \\
\hline $\begin{array}{l}\text { Sanjeev and } \\
\text { Anuradha } \\
(2012)^{(70)}\end{array}$ & Cross-sectional & Delhi, India & Children under 6 years & 462 & $\begin{array}{l}\text { Factors: Lack of education } \\
\text { Timing: Majority started before } 6 \text { months or after } 8 \text { months }\end{array}$ \\
\hline $\begin{array}{l}\text { Saxena and Kumar } \\
(2014)^{(61)}\end{array}$ & Cross-sectional & $\begin{array}{l}\text { Doiwala block, } \\
\text { Dehradun, India }\end{array}$ & $\begin{array}{l}\text { Mothers of children } \\
\text { under } 24 \text { months }\end{array}$ & 336 & $\begin{array}{l}\text { Diversity: Egg, vegetarian food } \\
\text { Frequency: } 31 \% \text { more than } 3 \text { times/d } \\
\text { Factors: Employment and lack of expressing, lack of knowledge, vomiting, child cries } \\
\text { Timing: } 13 \% \text { delayed, } 25 \% \text { early }\end{array}$ \\
\hline $\begin{array}{l}\text { Saxena and } \\
\quad \text { Kumari } \\
(2014)^{(77)}\end{array}$ & Cross-sectional & Doiwala block, India & $\begin{array}{l}\text { ASHA who consented to } \\
\text { participate and had a } \\
\text { child }\end{array}$ & 168 & $\begin{array}{l}\text { Diversity: Cow's milk, water, sugar, honey } \\
\text { Factors: Insufficient mother's milk }(55.4 \%) \text {, caesarean section }(20.2 \%) \text {, coercion from elders in the family to start } \\
\text { top milk, led to cessation of exclusive breast-feeding } \\
\text { Timing: Early for } 55 \%\end{array}$ \\
\hline
\end{tabular}




\section{Table 1 Continued}

\begin{tabular}{|c|c|c|c|c|c|}
\hline Study & Study type & Location & Population & $\begin{array}{c}\text { Sample } \\
\text { size }\end{array}$ & Adequacy of and factors influencing CFP \\
\hline $\begin{array}{l}\text { Senarath et al. } \\
(2012)^{(32)}\end{array}$ & Cross-sectional & $\begin{array}{l}\text { Bangladesh/Nepal/ } \\
\text { India/Sri Lanka/ } \\
\text { Pakistan }\end{array}$ & $\begin{array}{l}\text { Children aged between } \\
6 \text { and } 23 \text { months old }\end{array}$ & 15028 & $\begin{array}{l}\text { Diversity: MDD for } 6-23-\text { month-olds was } 15.2 \% \\
\text { Frequency: MMF was } 41.5 \% \\
\text { Factors: Lack of maternal education and lower household wealth, limited exposure to media, inadequate antenatal } \\
\text { care and lack of postnatal contacts by health workers } \\
\text { Timing: } 6-8 \text { months for } 55 \%\end{array}$ \\
\hline $\begin{array}{l}\text { Shahrawat and } \\
\text { Joon }(2013)^{(105)}\end{array}$ & Cross-sectional & Delhi, India & $\begin{array}{l}\text { Children aged } \\
0-24 \text { months }\end{array}$ & 5 & $\begin{array}{l}\text { Diversity: Grains, pulses, milk, fish, fruits } \\
\text { Factors: Better access to advice from health-care professionals }\end{array}$ \\
\hline $\begin{array}{l}\text { Sharan et al. } \\
(2001)^{(47)}\end{array}$ & Cross-sectional & Bangalore, India & $\begin{array}{l}\text { Farming women were } \\
\text { randomly selected }\end{array}$ & 306 & $\begin{array}{l}\text { Diversity: Ragi sari, rice with daal and ghee, vegetables, commercial baby food } \\
\text { Factors: Subsequent pregnancy, insufficient milk, child deemed old enough to wean }\end{array}$ \\
\hline $\begin{array}{l}\text { Sharma and } \\
\text { Sharma } \\
(2003)^{(82)}\end{array}$ & Cross-sectional & $\begin{array}{l}\text { Baijnath block of } \\
\text { Kangra district of } \\
\text { Himachal Pradesh, } \\
\text { India }\end{array}$ & $\begin{array}{l}\text { Mothers of children } \\
\text { under the age of } \\
2 \text { years }\end{array}$ & 100 & $\begin{array}{l}\text { Diversity: Kheer, dalia, dal, khichri, rice, fruits, vegetables, others } \\
\text { Factors: Knowledge, traditions, health status of mother, sanitation, education } \\
\text { Timing: } 70 \% \text { by } 4-6 \text { months }\end{array}$ \\
\hline $\begin{array}{l}\text { Shroff et al. } \\
\quad(2011)^{(74)}\end{array}$ & Cross-sectional & Andhra Pradesh, India & $\begin{array}{l}\text { Mothers of children } \\
\text { aged 3-15 months }\end{array}$ & 600 & $\begin{array}{l}\text { Factors: Autonomy of mother, tradition } \\
\text { Timing: } 24.9 \% \text { were taking foods or liquids other than breast milk at } 3-5 \text { months }\end{array}$ \\
\hline $\begin{array}{l}\text { Singh and Vaidya } \\
(2015)^{(106)}\end{array}$ & Cross-sectional & $\begin{array}{l}\text { Abalpur district of } \\
\text { Madhya Pradesh, } \\
\text { India }\end{array}$ & $\begin{array}{l}\text { Children aged } 6 \text { months } \\
\text { to } 3 \text { years }\end{array}$ & 300 & $\begin{array}{l}\text { Diversity: Cereals, pulses, millets, khichadi chawal, kudai bhat, latchaka, rejgeera ladoo } \\
\text { Factors: Working mothers were more likely to introduce complementary foods earlier than non-working mothers }\end{array}$ \\
\hline $\begin{array}{l}\text { Sinha and Pandey } \\
2000^{(83)}\end{array}$ & Cross-sectional & Bihar, India & $\begin{array}{l}\text { Mothers of children } \\
\text { under } 72 \text { months }\end{array}$ & 200 & $\begin{array}{l}\text { Diversity: Mandi, papaya, potatoes, rice, dhal, fish and fowl, rice } \\
\text { Factors: Lack of knowledge of mothers and health workers was a barrier to appropriate CF }\end{array}$ \\
\hline $\begin{array}{l}\text { Sinhababu et al. } \\
(2010)^{(84)}\end{array}$ & Cross-sectional & $\begin{array}{l}\text { Bankura town, West } \\
\text { Bengal, India }\end{array}$ & $\begin{array}{l}\text { Children aged } \\
0-23 \text { months }\end{array}$ & 647 & $\begin{array}{l}\text { Factors: Insufficient knowledge, inappropriate practices } \\
\text { Timing: } 56 \% \text { by } 6-8 \text { months }\end{array}$ \\
\hline $\begin{array}{l}\text { Sreedhara and } \\
\text { Banapurmath } \\
(2014)^{(65)}\end{array}$ & Cross-sectional & $\begin{array}{l}\text { Urban slum community } \\
\text { of central Karnataka, } \\
\text { India }\end{array}$ & $\begin{array}{l}\text { Infants aged } \\
\quad 9-12 \text { months }\end{array}$ & 100 & $\begin{array}{l}\text { Frequency: } 29 \% \text { were given } \mathrm{CF} \text { feeds less than } 3 \text { times/d } \\
\text { Timing: } 55 \% \text { between } 7-9 \text { months }\end{array}$ \\
\hline $\begin{array}{l}\text { Subbiah and } \\
\text { Jeganathan } \\
(2012)^{(81)}\end{array}$ & Cross-sectional & Delhi, India & $\begin{array}{l}\text { Postnatal mothers who } \\
\text { had a normal delivery }\end{array}$ & 405 & $\begin{array}{l}\text { Diversity: Sugar water and honey } \\
\text { Factors: Mothers need more support and information about breast-feeding and optimal times to begin CF }\end{array}$ \\
\hline $\begin{array}{l}\text { Tyagi and Bhan } \\
(2009)^{(75)}\end{array}$ & Cross-sectional & Hisar, India & $\begin{array}{l}\text { Mothers of children } \\
\text { aged } 0-60 \text { months }\end{array}$ & 380 & Factors: Maternal employment, lack of milk \\
\hline $\begin{array}{l}\text { Veena et al: } \\
(2010)^{(88)}\end{array}$ & Cohort & Mysore, India & $\begin{array}{l}\text { Mothers who delivered } \\
\text { babies at the } \\
\text { Holdsworth Memorial } \\
\text { Hospital }\end{array}$ & 514 & $\begin{array}{l}\text { Factors: Familial socio-economic status, maternal education, primiparity } \\
\text { Timing: Majority started at or after } 4 \text { months }\end{array}$ \\
\hline $\begin{array}{l}\text { Verma and Gupta } \\
(2015)^{(71)}\end{array}$ & Cohort & Uttar Pradesh, India & $\begin{array}{l}\text { Children aged below } \\
9 \text { months; vast } \\
\text { majority were under } \\
6 \text { months }\end{array}$ & 186 & $\begin{array}{l}\text { Diversity: Animal milk, cow's milk, porridge } \\
\text { Timing: Evidence of commencement at 3-6 months }\end{array}$ \\
\hline $\begin{array}{l}\text { Vyas et al. } \\
(2014)^{(48)}\end{array}$ & Cross-sectional & Uttarakhand, India & $\begin{array}{l}\text { Mothers with children } \\
\text { within } 3 \text { years of age } \\
\text { were included }\end{array}$ & 500 & $\begin{array}{l}\text { Diversity: Rice water (mand), coarse grains, jhingora, barley, maize, pulses, gahat, fruits, nuts } \\
\text { Factors: Lack of advice-seeking, cultural influences, education, socio-economic factors } \\
\text { Timing: } 52 \% \text { after } 6 \text { months }\end{array}$ \\
\hline Yasmin $(2008)^{(60)}$ & Cross-sectional & $\begin{array}{l}6 \text { different villages of } \\
\text { Chandaulia district, } \\
\text { Uttar Pradesh, India }\end{array}$ & $\begin{array}{l}\text { Mothers of children } \\
0-9 \text { months }\end{array}$ & 120 & $\begin{array}{l}\text { Diversity: Carrots, pumpkin, cauliflower, spinach, milk, buttermilk, potato, rice, pulses, porridge, kheer, banana } \\
\text { Frequency: } 90 \% \text { at } 6-9 \text { months } \\
\text { Factors: Perception of poor-quality breast milk } \\
\text { Timing: } 60 \% \text { at }<3 \text { months }\end{array}$ \\
\hline $\begin{array}{l}\text { Yousafzai et al. } \\
(2003)^{(85)}\end{array}$ & Cohort & Mumbai, India & $\begin{array}{l}\text { Carers of disabled and } \\
\text { non-disabled child }\end{array}$ & 41 & $\begin{array}{l}\text { Factors: Erroneous belief that a disability is curable takes the focus away from nutrition and its importance for the } \\
\text { well-being of children with disabilities. Unaffordability of food }\end{array}$ \\
\hline
\end{tabular}

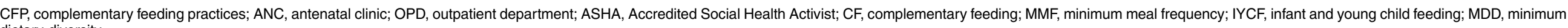
dietary diversity. 


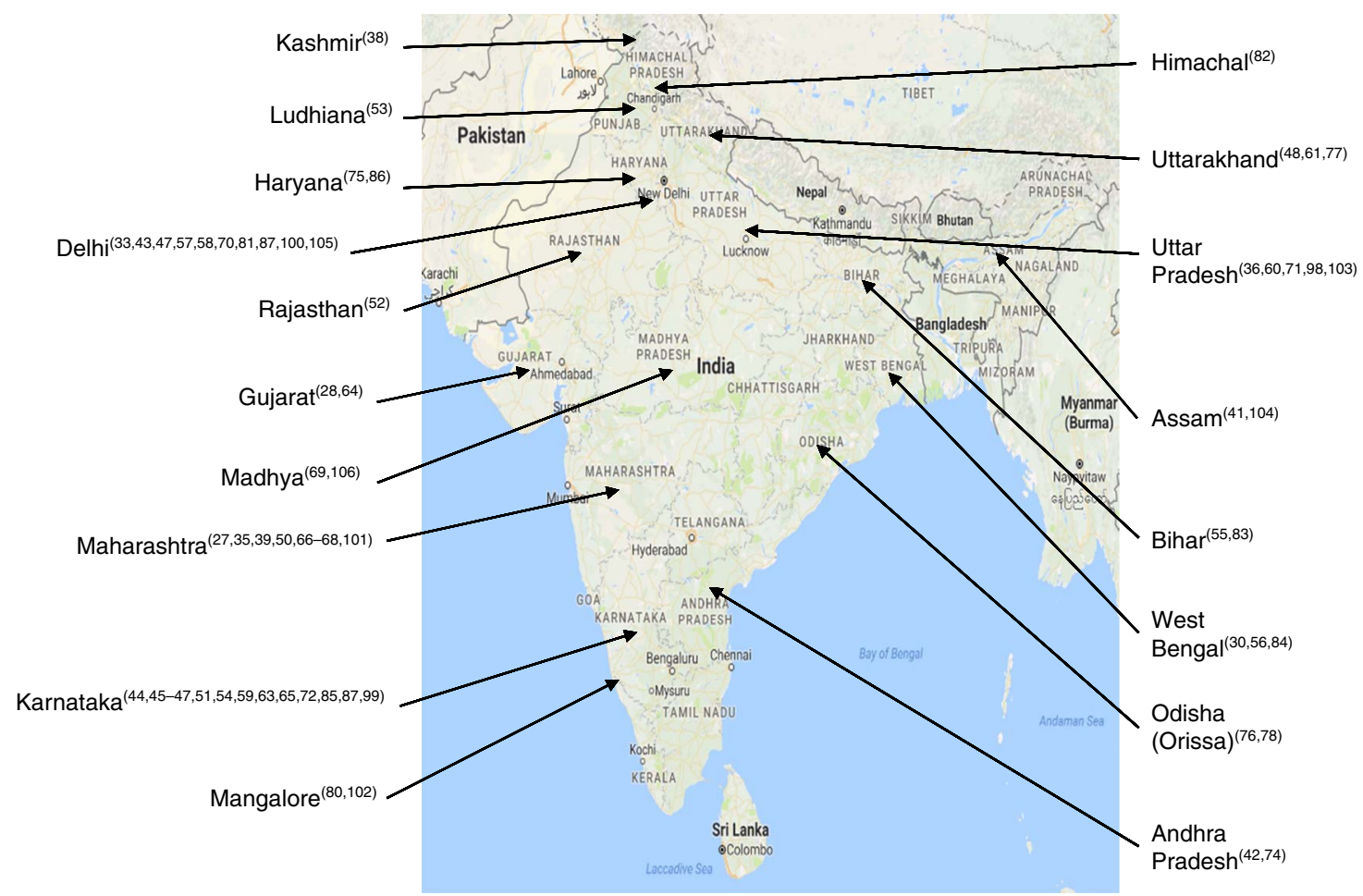

Fig. 2 (colour online) Location map of sixty-three studies included in the current systematic review (map courtesy of Google Maps; data (C) 2017 Google)

outcome for 6-23-month-olds ${ }^{(26-33)}$. In de Onis (WOE $\left.=\mathrm{M}\right)$, infants were fed a mean of $2 \cdot 8$ food groups at 6 months, rising to $5 \cdot 1$ at 24 months $^{(34)}$. Five other studies reported some information on diversity ${ }^{(35-39)}$.

Table 3 denotes a summary of all complementary food groups identified from the studies, categorized according to the WHO IYCF food groups defined above. Foods utilized for CF were identified in fifty-three included studies, of which nine had overall $\mathrm{WOE}=\mathrm{H}$ and forty-four had overall $\mathrm{WOE}=\mathrm{M}$.

Thirty-one studies identified 'grains, roots and tubers' being used for CFP. Legumes and nuts were used in twenty-nine, and twenty-six studies identified 'dairy products' (e.g. milk, cheese, yoghurt) being used. In contrast, 'eggs' were identified in twelve studies, 'flesh foods' (e.g. meat, fish, poultry and liver/organ meats)' in ten studies, 'vitamin A-rich fruits and vegetables' in eight studies and 'other fruits and vegetables' in twenty-two studies.

Bentley et al. (WOE $=\mathrm{H})$ found that grains were consumed by $63.8 \%$ of infants in the past $24 \mathrm{~h}^{(27)}$. In Fazilli et al. $(\mathrm{WOE}=\mathrm{M}), \mathrm{Neog}$ and Baruah $(\mathrm{WOE}=\mathrm{M})$ and Meshram et al. (WOE $=\mathrm{H})$, cereals were also widely used $^{(40-42)}$. In Katara et al. $(\mathrm{WOE}=\mathrm{M})$, cereals were the most frequently used food group, by $96 \%$ of infants ${ }^{(37)}$. In contrast, in Kapur et al. (WOE=M) cereal intake in an urban slum in Delhi was noted as grossly inadequate ${ }^{(43)}$. $R a g i$, a traditional Indian grain, was identified in four studies as a common cereal type utilized in South India ${ }^{(44-47)}$.

The use of 'other fruits and vegetables', namely fruits and vegetables not specified as vitamin A-rich, varied across India, from $95.4 \%$ among study populations in rural Andhra Pradesh to $1.45 \%$ in Uttarakhand when given alone ${ }^{(42,48)}$. Interestingly, in Garg and Chadha (WOE = M) in rural India, fruits and vegetables were excluded from an infant's diet despite being part of the family diet due to beliefs that infants could not tolerate spice-cooked fruits and vegetables ${ }^{(36)}$. In Vyas et al. $(\mathrm{WOE}=\mathrm{M})$, seasonal fruits such as guava and citrus were introduced before the addition of staples (e.g. cereals, rice), with gross undernutrition noted in the study population ${ }^{(48)}$.

In the WHO Multicentre Growth Reference Study, less than $11 \%$ of children were noted to consume flesh foods ${ }^{(34)}$. In an affluent Delhi district, Bhandari et al. (WOE $=\mathrm{M}$ ) found that only $2.4 \%$ of infants consumed non-vegetarian foods despite $57.5 \%$ of their families being non-vegetarian ${ }^{(49)}$. Consumption of Fe-rich or Fe-fortified foods (e.g. flesh foods) was poorly reported. Kapur et al. (WOE =M) found that children consumed only $46 \%$ of the RDA for Fe in their diets, and Pashricha et al. $(\mathrm{WOE}=\mathrm{M})$ found that delayed $\mathrm{CF}$ increased the risk of low dietary Fe intake ${ }^{(43,44)}$. Bentley et al. $(\mathrm{WOE}=\mathrm{H}$ ) found that $15 \%$ of $6-23$-month-olds consumed Fe-rich foods, which is similar to the $12 \cdot 1 \%$ reported by Aguayo et al. $(\mathrm{WOE}=\mathrm{H})^{(27,50)}$.

Regarding commercial complementary foods, Sharan et $a l .(\mathrm{WOE}=\mathrm{M})$ and Samuel et al. $(\mathrm{WOE}=\mathrm{H})$ noted use of commercial foods ${ }^{(47,51)}$. Cerelac was the most frequently mentioned commercial food ${ }^{(41,46,52-54)}$. Additionally, Ananda Kumar et al. (WOE=M), Lingam et al. $(\mathrm{WOE}=\mathrm{H})$ and Chhabra et al. $(\mathrm{WOE}=\mathrm{M})$ mentioned Farex, and Narayanappa et al. mentioned Nestum ${ }^{(46,52-54)}$. 
Table 2 Weight of evidence awarded to each study in the current systematic review

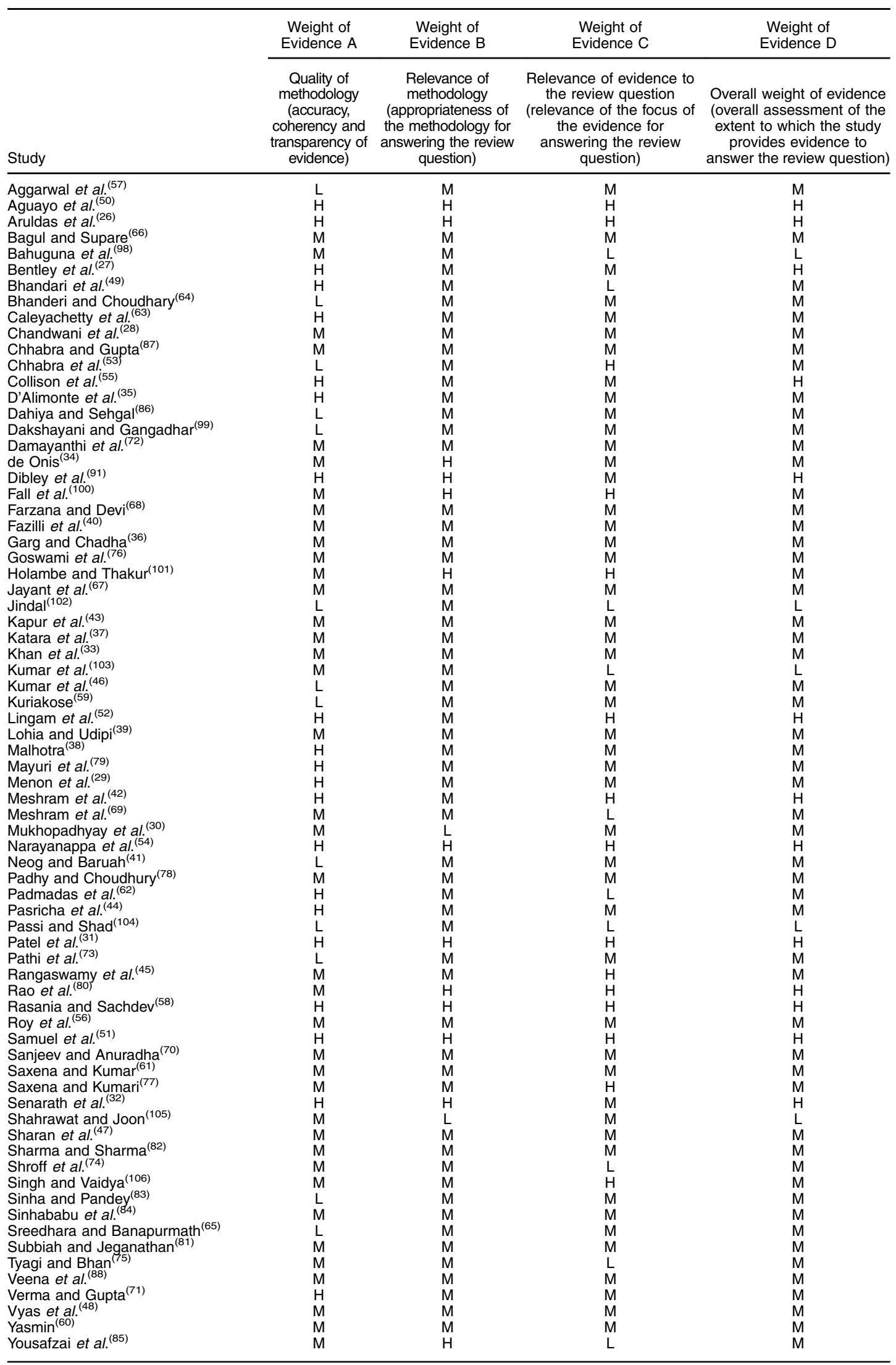

L, low; M, medium; $\mathrm{H}$, high. 
Table 3 Foods utilized for complementary feeding in India, categorized into WHO food groups

\begin{tabular}{ll}
\hline WHO classified food groups & Number of studies and references \\
\hline Grains, roots and tubers & Thirty-one studies $^{(26-29,31,34,36,37,40,42,43,45-48,50,52-54,56,60,64,68,71,79,80,82-84,86,106)}$ \\
Legumes and nuts & Twenty-nine studies $^{(26-29,31,34,37,40,43,45-48,50,52-54,56,60,64,68,69,79,80,82-84,86,106)}$ \\
Dairy products (e.g. milk, cheese, yoghurt) & Twenty-six studies $^{(26-28,31,34,36,37,41-43,45-47,49,50,53,54,60,64,68,71,77,79,82,84,86)}$ \\
$\begin{array}{l}\text { Flesh foods (e.g. meat, fish, poultry and } \\
\quad \text { liver/organ meats) }\end{array}$ & Eleven studies $^{(26-29,34,43,49,50,54,55,83)}$ \\
Other fruits and vegetables & Twenty-two studies $^{(26-28,31,33,36,37,40,41,43,47-50,53-55,60,64,68,83,86)}$ \\
Vitamin A-rich fruits and vegetables (e.g. pumpkin) & Eight studies $^{(27,28,31,33,42,50,60,61)}$ \\
Eggs & Twelve studies $^{(26-29,31,34,36,49,50,54,55,61)}$ \\
\hline
\end{tabular}

Chhabra et al. also mentioned Nutramul ${ }^{(53)}$. In Sharan et al. only $15 \%$ of infants were given commercial complementary food, with use concentrated among the highest socio-economic group ${ }^{(47)}$, in keeping with Lingam et al. $(\mathrm{WOE}=\mathrm{H})$ who noted higher utilization rates in urban compared with rural areas ${ }^{(52)}$.

Generally, micronutrient intake was not discussed in the included studies. In Pasricha et al. (WOE=M), 66\% of children were found to be deficient in at least one micronutrient, with micronutrient deficiencies particularly common in those who breast-fed longer ${ }^{(44)}$. The high use of grains and legumes by included infants may be beneficial, as Menon et al. found that intakes of these foods were associated with positive anthropometric outcomes relative to higher-nutrient foods like eggs or flesh foods ${ }^{(29)}$.

\section{Meal frequency}

Meal frequency was explored in twenty-one studies $^{(26,27,30,31,33,34,36-39,42,46,50,51,55-61)}$. In ten studies, MMF was attained by between 25 and $50 \%$ of the study population $^{(27,31,33,37,38,46,57-59,61)}$. In contrast, between 50 and $96 \%$ of the population achieved MMF in seven studies ${ }^{(26,30,36,39,42,50,60)}$. Seven included studies had overall $\mathrm{WOE}=\mathrm{H}$ and fourteen had overall $\mathrm{WOE}=\mathrm{M}$.

Senarath et al. $(\mathrm{WOE}=\mathrm{H})$ noted that the rate of MMF was $42 \%$ in children aged 6-23 months ${ }^{(32)}$. Patel et al. (WOE = $\mathrm{H})$ and Khan et al. (WOE $=\mathrm{M}$ ) observed MMF in 41.5 and $48.6 \%$ of children, respectively ${ }^{(31,33)}$. In contrast, Chandwani et al. $(\mathrm{WOE}=\mathrm{M})$ noted that $96 \%$ of breast-fed children were fed at least the minimum number of times recommended ${ }^{(28)}$.

Malhotra $(\mathrm{WOE}=\mathrm{M})$ noted a correlation between education and meal frequency in infants aged 918 months $^{(38)}$. Finally, Lohia and Udipi $(\mathrm{WOE}=\mathrm{M})$ noted that male infants tended to have a higher feeding frequency than female infants ${ }^{(39)}$.

\section{Timing of introducing complementary feeding}

Table 4 denotes a summary of timing when CF was most commonly introduced across the fifty-nine included studies that investigated timing. The most common age for the introduction of $\mathrm{CF}$ was between 6 and 9 months (twenty-nine studies), followed by 3 to 6 months (twentytwo studies). Four studies noted that CF was started between 9 and 12 months for the majority of infants, while one study noted that $\mathrm{CF}$ was started at an age younger than 3 months for most infants. Twelve studies had overall $\mathrm{WOE}=\mathrm{H}$ and forty-seven had overall $\mathrm{WOE}=\mathrm{M}$.

CF was noted to be delayed among children particularly in central and eastern India ${ }^{(62)}$. Inappropriate timing of initiation of $\mathrm{CF}$ was noted in both urban and rural regions of India, with timely $\mathrm{CF}$ achieved by as low as $3.5 \%$ and as late as over 1 year of age ${ }^{(47,49,56,63)}$. In ten out of fifteen studies in urban areas, the majority of children started CF at 6-9 months $(27,33,37,50,53,55,56,65,80,84)$. Eight out of eighteen studies in rural areas noted that CF started during 6-9 months of age $26,28,42,48,52,61,67,69)$, and seven out of eighteen noted that CF initiated at 3-6 months ${ }^{(45,54,63,68,71,72,74)}$

In addition, Yasmin $(\mathrm{WOE}=\mathrm{M})$ noted that $\mathrm{CF}$ was initiated as early as 1 week $^{(60)}$. However, in Mukhopadhyay et al. (WOE $=\mathrm{M}), \mathrm{CF}$ timing was noted to be inappropriately early in $12.5 \%$ of the study population in West Bengal slums ${ }^{(30)}$. Similar findings were also noted in Goswami et al. (WOE $=\mathrm{M}$ ), where only $13 \cdot 2 \%$ of the infants were introduced to $\mathrm{CF}$ at the age of 4-6 months ${ }^{(76)}$, and in Roy et al. $(\mathrm{WOE}=\mathrm{M})$ in an urban slum in Kolkata where $72 \%$ of infants were given $\mathrm{CF}$ at 6 months ${ }^{(56)}$.

\section{Sources of advice for feeding}

Twenty-seven studies described advice providers for CFP, of which nine had overall $\mathrm{WOE}=\mathrm{H}$ and eighteen had overall $\mathrm{WOE}=\mathrm{M}$. The commonest source of feeding advice were health-care professionals, including doctors, auxillary nurse midwives, lady health visitors and anganwadi health workers, usually at antenatal visits or during immunizations (twenty-one studies ${ }^{(26,35,38,45,46,50,51,53-57,60,61,64,66,68,77-80)}$ ). The next most common source of advice was a family member, usually the grandmother or mother-in-law (eleven studies $^{(26,35,45,46,48,52,54,55,60,67,81)}$, with nine further studies specifically mentioning elders ${ }^{(35,40,42,45,51,61,66,77,79)}$. Further sources of feeding advice were the media (four studies $\left.{ }^{(31,35,38,45)}\right)$ and friends (three studies $\left.{ }^{(45,56,60)}\right)$.

\section{Factors associated with complementary feeding practices}

We identified numerous factors that influenced CFP. These are summarized in Table 5 as either a barrier or a promoter, and sub-categorized as acting at either family or organizational level. Due to conflicting study findings, 
Table 4 Timing of introduction of complementary feeding in India

\begin{tabular}{ll}
\hline Infant age & Number of studies and references \\
\hline$<3$ months & One study ${ }^{(60)}$ \\
$3-6$ months & Twenty-two studies $(29,34,45,46,49,51,54,58,59,62-64,66,68,71,72,77,78,82,86,88,106)$ \\
$6-9$ months & Twenty-nine studies $(26-28,30-33,35,37,38,40-42,48,50,52,53,56,65,67,69,76,80,84,86,87,91,99,101)$ \\
$9-12$ months & Four studies $(40,57,73,100)$ \\
$>12$ months & Zero studies \\
\hline
\end{tabular}

factors may appear as both a barrier and a promoter. Twenty-four promoters and thirty barriers influencing CFP were identified. Promoters and barriers were further divided into factors influencing at the family and organizational level. In total, fifty-five studies identified factors associated with CF practices, of which twelve had overall $\mathrm{WOE}=\mathrm{H}$ and forty-three had overall $\mathrm{WOE}=\mathrm{M}$.

\section{Barriers}

Thirty-five studies identified barriers at the organizational level. Barriers were: cultural influences, employment, food insecurity, gender, inadequate antenatal care, lack of knowledge on optimal CFP, lack of media exposure, lack of parental education, location: Northern India and West India, focus on disability, low literacy, poor sanitation, poverty, birth in a public hospital and price of food. The most commonly cited barrier at the organizational level was cultural influences $(40,41,45,48,51,53,55,57,61,64,74,77-79,82)$. Infant feeding practices in India appear to be strongly influenced by elderly women such as the mother-in$\operatorname{law}^{(48,67)}$.

Thirty-one studies identified barriers at the family level. Barriers were: caesarean section, child's age, concern about weight gain, crying infant, difficulty feeding child, inadequate breast milk production, lack of support, maternal age, maternal nutrition status, mothers from joint families, recent illness, religion, siblings, subsequent pregnancy and primiparity. The most commonly cited barriers at the family level were lack of knowledge on optimal $\mathrm{CFP}^{(26,40,48,53,61,67,77,81-85)}$ and inadequate breast milk production ${ }^{(45,47,54,60,61,75,77)}$.

\section{Promoters}

Thirty-two studies identified promoters at the organizational level. Promoters were: advice from a health-care professional, birth within a government institute, certain caste or tribe, education of parent, effective antenatal care, family support, Hindu mothers, literacy status of mother, location: north-eastern, southern or western, media exposure, social support group, socio-economic status, support system at work and wealth. The most commonly cited promoters at the organizational level were education of parent $(26,29,31,36,39,41,42,44,48,63,64,68,73)$, literacy status of mother $^{(37,66,68,72,80,86)}$ and wealth ${ }^{(26,36,52,87,88)}$.

Twelve studies identified promoters at the family level. Promoters were: acknowledged importance of maternal health, advice seeking, autonomy of mother, BMI of mother, delivery with doctor present, high birth order, knowledge of optimal CFP, mother who works from home, older age at marriage and valuing nutrition. The most commonly cited promoter at the family level was knowledge of optimal $\mathrm{CF}^{(35,56,67,78)}$.

\section{Discussion}

To our knowledge, the present is the first systematic review to assess CFP in India. We identified that in many SA families in India, WHO IYCF standards on minimum dietary diversity, meal frequency and timing of introducing CF were not being met.

\section{Implications of key findings}

Legumes, rice, wheat and cereals appear to be the mainstay of complementary foods in Southern India. While this is in keeping with other low- and middle-income countries, these foods have low nutrient density and mineral bioavailability, and the use of other food groups is essential to satisfy the nutrient and mineral requirements of infants ${ }^{(89)}$. Consumption of dietary Fe was infrequently mentioned except in the context of flesh foods, and was inadequate, considering that Fe has such an important role in infant health ${ }^{(43,44)}$.

Dietary diversity was found to be inadequate in almost all groups studied, with MDD achieved in only 6 to $33 \%$ of 6-23-month-olds. Some have argued for use of media sources to influence this, with further research and interventions needed ${ }^{(39)}$.

It was found that MMF was not met by the majority of the populations sampled. Educational interventions may be useful to improve MMF going forward; Collison et al. found that frequency of feeds increased when families were given a feeding toolkit ${ }^{(55)}$. In a previous review, educational interventions were also shown to be effective ${ }^{(90)}$. Further research is required to uncover why MMF is so rarely met by caregivers.

The majority of studies found that CF was started during months 6-9 of life, with most studies noting limited maternal awareness on recommended CFP. By improving antenatal care and education on caring for an infant alongside decreasing barriers faced by mothers when restarting employment, optimal timing of CF may improve. Mass communication using ICT and mobile apps is a strategy that has been advocated by the Ministry of Women and Child Development ${ }^{(92)}$, and could be used to disseminate information on this topic. 
Table 5 Factors influencing complementary feeding practices (CFP) in India

\begin{tabular}{|c|c|c|c|}
\hline \multicolumn{4}{|c|}{ Family level } \\
\hline Promoters & Number of studies and references & Barriers & Number of studies and references \\
\hline $\begin{array}{l}\text { Knowledge of optimal CFP } \\
\text { Autonomy of mother } \\
\text { Older age at marriage } \\
\text { Valuing nutrition } \\
\text { BMI of mother } \\
\text { Delivery with doctor present } \\
\text { High birth order } \\
\text { Acknowledged importance of maternal health } \\
\text { Advice-seeking } \\
\text { Mother who works from home }\end{array}$ & $\begin{array}{l}\text { Four studies } \\
\text { Two studies } \\
\text { Two studies }^{(29,82,56,67,78)} \\
\text { Two studies } \\
\text { One study }(39,78) \\
\text { One study } \\
\text { One study } \\
\text { On } \\
\text { One study }(35) \\
\text { One study } \\
\text { One study } \\
{ }^{(38)}\end{array}$ & $\begin{array}{l}\text { Lack of knowledge of optimal CFP } \\
\text { Inadequate breast milk production } \\
\text { Siblings } \\
\text { Recent illness } \\
\text { Difficulty feeding child } \\
\text { Crying infant } \\
\text { Lack of support } \\
\text { Maternal age } \\
\text { Religion } \\
\text { Caesarean section } \\
\text { Child's age } \\
\text { Concern about weight gain } \\
\text { Maternal nutrition status } \\
\text { Mothers from joint families } \\
\text { Primiparity } \\
\text { Subsequent pregnancy }\end{array}$ & 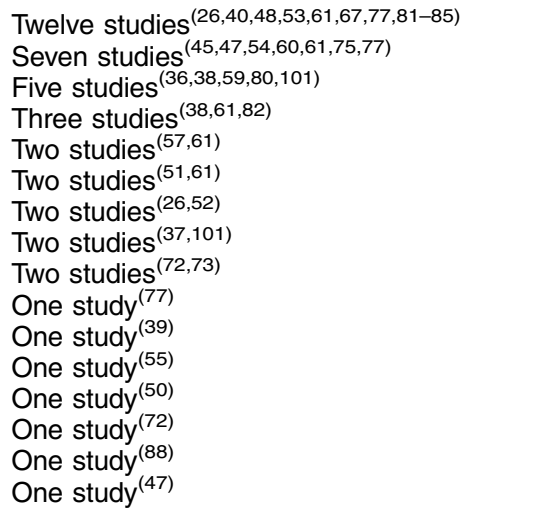 \\
\hline \multicolumn{4}{|c|}{ Organizational level } \\
\hline Promoters & Number of studies and references & Barriers & Number of studies and references \\
\hline Education of parent & $\begin{array}{l}\text { Fourteen } \\
\text { studies } \\
(26,29,31,36,39,41,42,44,48,63,64,68,73,88)\end{array}$ & Cultural influences & $\begin{array}{l}\text { Fifteen } \\
\quad \text { studies } \\
(40,41,45,48,51,54,55,57,61,64,74,77-79,82)\end{array}$ \\
\hline Literacy status of mother & Six studies ${ }^{(37,66,68,72,80,86)}$ & Poverty & Six studies $(29,32,50,52,69,78)$ \\
\hline Wealth & Five studies $(26,36,52,87,88)$ & Lack of parental education & Six studies ${ }^{(32,57,62,70,82,101)}$ \\
\hline Socio-economic status & Five studies $(36,54,63,72,86)$ & Low literacy & Five studies $(29,32,66,69,79)$ \\
\hline Media exposure & Four studies $(26,38,63,91)$ & Employment & Four studies $(51,61,75,86)$ \\
\hline Social support group & Three studies ${ }^{(35,67,72)}$ & Gender & Four studies ${ }^{(37,39,43,87)}$ \\
\hline Advice from a health-care professional & Three studies $(38,56,70)$ & Poor sanitation & Three studies ${ }^{(50,82,88)}$ \\
\hline Effective antenatal care & Three studies ${ }^{(26,64,91)}$ & Inadequate antenatal care & Two studies ${ }^{(31,32)}$ \\
\hline Location & Three studies ${ }^{(62,64,91)}$ & Food insecurity & Two studies ${ }^{(44,82)}$ \\
\hline Certain caste or tribe & Two studies $(42,69)$ & Price of food & Two studies $(55,85)$ \\
\hline Support system at work & One study ${ }^{(77)}$ & Focus on disability & One study ${ }^{(85)}$ \\
\hline Family support & One study ${ }^{(81)}$ & Birth in a public hospital rather than a private hospital & One study ${ }^{(80)}$ \\
\hline Hindu mothers & One study ${ }^{(63)}$ & Location: Northern India, West India & One study ${ }^{(31)}$ \\
\hline Birth within a government institute & One study ${ }^{(87)}$ & Lack of media exposure & One study(32) \\
\hline
\end{tabular}


Of the studies that identified sources of feeding advice, health-care professionals were the most commonly cited. Antenatal check-ups especially were a popular time for feeding advice to be given to mothers by health-care professionals $^{(31,32,38,58,76,84)}$. Family members, particularly a mother-in-law or grandmother, were also very commonly cited sources of feeding advice. However, the advice given by them is often inappropriate. Saxena and Kumar noted that some female elders insisted mothers only started CF after 1 year $^{(61)}$. There is a suggestion that family members can adversely influence mothers through conveying traditional beliefs, for example that colostrum is 'dirty', and that children cannot tolerate animal-based proteins until 18 months of age ${ }^{(45,55,67)}$. Similar advice may also be conveyed by friends and peer groups. Media, including radio, newspapers and magazines, was an important but less commonly cited source of advice. Malhotra found that increased frequency of listening to the radio or of reading newspapers and magazines carried an increased likelihood of mothers having better feeding practices $^{(38)}$.

Several studies identified cultural norms introduced by female elders that are barriers to appropriate CFP, such as preferential treatment of male infants. It is therefore key that opinion leaders are equally targeted in any intervention to improve CFP in communities. Studies by Senarath et $a l^{(32)}$ and Dewey and Brown ${ }^{(93)}$ noted the effectiveness of systematic, participatory and coordinated approaches to improve CFP through peers and community facilitators, in keeping with UNICEF guidance on applying best practices and design in interventions ${ }^{(94)}$.

We hope our identification of barriers and promoters will provide inspiration for further interventions to improve CFP. Existing interventions in India have been educational in nature, including counselling ${ }^{(95)}$, resulting in increased energy intake and length; and education in complementary and responsive feeding ${ }^{(96)}$, resulting in increased energy intake and reduced stunting. The Lancet 2008 series on maternal and child nutrition included a piece on successful interventions across countries ${ }^{(97)}$.

\section{Strengths and limitations}

The strengths of our systematic review are derived by searching a large number of databases utilizing very broad search strings, performing an updated search in June 2016, and having two reviewers undertake study selection, data extraction and quality assessment.

Key limitations include exclusion of: (i) papers which focused solely on children over 2 years of age, where CFP described in their younger years may have been missed; (ii) papers published before the year 2000 at full-text review; and (iii) papers not published in English, which would have added to the diversity of CFP described.

In several studies where there was overlap between children under and over 2 years and/or SA by Indian, Pakistani and Bangladeshi origin, CFP described and attributed to the whole study population may be incorrect. Furthermore, we did not assess the quantities of the foods used, only the frequency with which they appeared in the studies.

While we excluded interventional studies that may have described CFP in their study population, this is unlikely to be the primary focus of such studies and therefore unlikely to have affected our systematic review significantly. Additionally, if we had included strict exclusion criteria for study design, this may have meant there was less of a need to exclude studies due to low overall WOE rating; however, on the other hand, we may have missed some useful studies by being more prescriptive.

Regarding bias, while we attempted to contact numerous authors to identify relevant grey literature for our review, due to the breadth and depth of the field of nutritional research, this is unlikely to have been exhaustive and publication bias is likely to be present. Additionally, the vast majority of studies ( $n$ 64) were crosssectional, commonly using recall methods, with only seven cohort studies. This may mean reported results are biased towards time points when it is convenient to collect single sets of data, such as during medical visits.

\section{Conclusion}

Despite adoption of the WHO IYCF guidelines, inadequate CFP remain in SA communities across India. While India has made giant strides in decreasing child mortality over the last two decades, more must be done to improve CFP to further this aim. The present systematic review has highlighted CFP and the factors that influence them, providing knowledge of current behaviours; we recommend this information be used for context-tailored interventions that can be assessed and adopted according to their achievements.

\section{Acknowledgements}

Acknowledgements: The authors would like to thank Jia Ying Kuah, Anika Sharmila, Lucy Stephenson, Melanie Flury, Taimur Shafi, Atul Singhal, Rani Chowdhary, Charlotte Hamlyn-Williams, Prerna Bhasin and Conor Fee for assisting in the systematic review. Financial support: This research received no specific grant from any funding agency in the public, commercial or not-for-profit sectors. L.M. is funded by a National Institute for Health Research (NIHR) Doctoral Research Fellowship (grant number DRF-2014-07-005); S.A. is funded by the NIHR Collaboration for Leadership in Applied Health Research and Care (CLAHRC) North Thames at Bart's Health NHS Trust; and M.L. is partly supported by the NIHR CLAHRC North Thames at Bart's Health NHS Trust. NIHR had no role in the design, analysis or writing of this article. Conflict of 
interest: The authors declare that they have no competing interests. Authorship: L.M., R.L. and M.L. conceived and participated in the design of the study. L.M., A.P., A.D., C.M., A.R., A.L. and S.A. coordinated and undertook the review. All authors performed the data interpretation and contributed equally to write the draft, read and approved the final manuscript. Ethics of buman subject participation: Not applicable.

\section{References}

1. Black RE, Victora CG, Walker SP et al. (2013) Maternal and child undernutrition and overweight in lowincome and middle-income countries. Lancet 382, 427-451.

2. UNICEF (2009) Tracking Progress on Child and Maternal Nutrition: A Survival and Development Priority. New York: UNICEF.

3. World Health Organization (2016) Appropriate complementary feeding. http://www.who.int/elena/titles/ complementary_feeding/en/ (accessed October 2016).

4. Davies-Adetugbo AA \& Adetugbo K (1997) Effect of early complementary feeding on nutritional status in term infants in rural Nigeria. Nutr Health 12, 25-31.

5. Semahegn A, Tesfaye G \& Bogale A (2014) Complementary feeding practice of mothers and associated factors in Hiwot Fana specialized hospital, eastern Ethiopia. Pan Afr Med J 18, 143.

6. Macharia CW, Kogi-Makau W \& Muroki NM (2004) Dietary intake, feeding and care practices of children in Kathonzweni division, Makueni district, Kenya. East Afr Med J 81, 402-407.

7. Stewart CP, Iannotti L, Dewey KG et al. (2013) Contextualising complementary feeding in a broader framework for stunting prevention. Matern Child Nutr 9, 27-45.

8. Dewey KG (2016) Reducing stunting by improving maternal, infant and young child nutrition in regions such as South Asia: evidence, challenges and opportunities. Matern Child Nutr 12, 27-38.

9. Avula R, Raykar N, Menon P et al. (2016) Reducing stunting in India: what investments are needed? Matern Child Nutr 12, 249-252.

10. Ramji S (2009) Impact of infant \& young child feeding \& caring practices on nutritional status \& health. Indian J Med Res 130, 624-626.

11. Engle PL (2002) Infant feeding styles: barriers and opportunities for good nutrition in India. Nutr Rev $60,5 \mathrm{Pt}$ 2, S109-S114.

12. Senarath U \& Dibley MJ (2012) Complementary feeding practices in South Asia: analyses of recent national survey data by the South Asia Infant Feeding Research Network. Matern Child Nutr 8, 5-10.

13. Gragnolati M, Shekar M, Das Gupta M et al. (2005) India's Undernourished Children: A Call for Reform and Action. Washington, DC: The World Bank.

14. World Health Organization (2010) Indicators for Assessing Infant and Young Child Feeding Practices. Geneva: WHO.

15. Manikam L, Sharmila A, Dharmaratnam A et al. (2017) Systematic review of infant and young child complementary feeding practices in South Asian families: the Pakistan perspective. Public Health Nutr (Epublication ahead of print version, doi:10.1017/S1368980017002956).

16. Manikam L, Robinson A, Kuah JY et al. (2017) A systematic review of complementary feeding practices in South Asian infants and young children: the Bangladesh perspective. BMC Nutr 3, 56.

17. Porta M (2014) A Dictionary of Epidemiology, 6th ed. New York: Oxford University Press.
18. National Institute of Clinical Excellence (n.d.) NICE Glossary. https://www.nice.org.uk/glossary (accessed November 2016).

19. Centre for Reviews and Dissemination (2009) Systematic Reviews: CRD's guidance for undertaking reviews in health care. https://www.york.ac.uk/media/crd/Systematic_Reviews. pdf (accessed November 2016).

20. Dixon-Woods M, Agarwal S, Jones D et al. (2005) Synthesising qualitative and quantitative evidence: a review of possible methods. $J$ Health Serv Res Policy 10, $45-53$.

21. Collins JA \& Fauser BCJM (2005) Balancing the strengths of systematic and narrative reviews. Hum Reprod Update 11, 103-104.

22. Popay J, Roberts H, Sowden A et al. (2006) Guidance on the Conduct of Narrative Synthesis in Systematic Reviews: A Product from the ESRC Methods Programme. http://www.lancaster.ac.uk/shm/research/nssr/research/ dissemination/publications/NS_Synthesis_Guidance_v1.pdf (accessed October 2017).

23. Oxford Dictionaries (2017) Definition of barrier in English. https://en.oxforddictionaries.com/definition/barrier (accessed October 2017).

24. Oxford Dictionaries (2017) Definition of promoter in English. https://en.oxforddictionaries.com/definition/ promoter (accessed October 2017).

25. Gough D (2007) Weight of Evidence: a framework for the appraisal of the quality and relevance of evidence. Res Pap Educ 22, 213-228.

26. Aruldas K, Khan MM, Hazra A et al. (2010) Increasing appropriate complementary feeding in rural Uttar Pradesh. J Fam Welf 56, 51-56.

27. Bentley A, Das S, Alcock G et al. (2015) Malnutrition and infant and young child feeding in informal settlements in Mumbai, India: findings from a census. Food Sci Nutr 3 , 257-271.

28. Chandwani H, Prajapati A, Rana B et al. (2015) Assessment of infant and young child feeding practices with special emphasis on IYCF indicators in a field practice area of rural health training centre at Dabhoda, Gujarat, India. Int J Med Sci Public Health 4, 1414-1419.

29. Menon P, Bamezai A, Subandoro A et al. (2015) Ageappropriate infant and young child feeding practices are associated with child nutrition in India: insights from nationally representative data. Matern Child Nutr 11, $73-87$.

30. Mukhopadhyay DDK, Sinhababu A, Saren AAB et al. (2013) Association of child feeding practices with nutritional status of under-two slum dwelling children: a community-based study from West Bengal, India. Indian J Public Health 57, 169-172.

31. Patel A, Pusdekar Y, Badhoniya N et al. (2012) Determinants of inappropriate complementary feeding practices in young children in India: secondary analysis of National Family Health Survey 2005-2006. Matern Child Nutr 8, Suppl. 1, 28-44.

32. Senarath U, Agho KE, Akram DES et al. (2012) Comparisons of complementary feeding indicators and associated factors in children aged 6-23 months across five South Asian countries. Matern Child Nutr 8, Suppl. 1, 89-106.

33. Khan AAM, Kayina P, Agrawal P et al. (2012) A study on infant and young child feeding practices among mothers attending an urban health center in East Delhi. Indian $J$ Public Health 56, 301-304.

34. de Onis M (2006) Complementary feeding in the WHO Multicentre Growth Reference Study. Acta Paediatr Suppl 450, 27-37.

35. D'Alimonte MR, Deshmukh D, Jayaraman A et al. (2016) Using positive deviance to understand the uptake of optimal infant and young child feeding practices by 
mothers in an urban slum of Mumbai. Matern Child Health J 20, 1133-1142.

36. Garg A \& Chadha R (2009) Index for measuring the quality of complementary feeding practices in rural India.J Health Popul Nutr 27, 763-771.

37. Katara PS, Patel SV, Kantharia SL et al. (2013) Study on feeding practices among children 6 months to 2 years and its effect on their nutritional status in urban slums. Natl $J$ Community Med 4, 475-478.

38. Malhotra N (2013) Inadequate feeding of infant and young children in India: lack of nutritional information or food affordability? Public Health Nutr 16, 1723-1731.

39. Lohia N \& Udipi SA (2014) Infant and child feeding index reflects feeding practices, nutritional status of urban slum children. BMC Pediatr 14, 290.

40. Fazilli A, Imtiyaz A, Iqbal M et al. (2011) Infant feeding practices of multiparous women attending the antenatal clinic in a tertiary care hospital. Int J Med Public Health 1, 47-50.

41. Neog N \& Baruah DK (2012) Prevalent infant feeding practices of infants among the missing tribes of Jorhat district, Assam. Food Sci Res J 3, 9-13.

42. Meshram II, Laxmaiah A, Venkaiah K et al. (2012) Impact of feeding and breastfeeding practices on the nutritional status of infants in a district of Andhra Pradesh, India. Natl Med J India 25, 201-206.

43. Kapur D, Sharma S \& Agarwal KN (2005) Dietary intake and growth pattern of children 9-36 months of age in an urban slum in Delhi. Indian Pediatr 42, 351-356.

44. Pasricha S-RR, Shet ASS, JFF Black et al. (2011) Vitamin B-12, folate, iron, and vitamin A concentrations in rural Indian children are associated with continued breastfeeding, complementary diet, and maternal nutrition. $A m$ J Clin Nutr 94, 1358-1370.

45. Rangaswamy K, Kumar A \& Kumar HV (2013) Sociocultural factors influencing infant feeding practices in rural Tumkur. Res I Pharmaceut Biol Chem Sci 4, 204-212.

46. Ananda Kumar TS, Rangaswamy KB \& Viswanatha Kumar HM (2013) Weaning practices in rural Tumkur. Curr Pediatr Res 17, 115-117.

47. Sharan S, Kumari SPS \& Nagabhushanam K (2001) Status of sericulture farm women and infant feeding habits. Mysore J Agric Sci 35, 351-356.

48. Vyas S, Kandpal SD, Semwal J et al. (2014) Trends in weaning practices among infants and toddlers in a hilly terrain of a newly formed state of India. Int J Prev Med $\mathbf{5}$, 741-748.

49. Bhandari N, Bahl R, Taneja S et al. (2002) Growth performance of affluent Indian children is similar to that in developed countries. Bull World Health Organ 80, 189-195.

50. Aguayo VM, Nair R, Badgaiyan N et al. (2016) Determinants of stunting and poor linear growth in children under 2 years of age in India: an in-depth analysis of Maharashtra's comprehensive nutrition survey. Matern Child Nutr 12, 121-140.

51. Samuel TM, Thomas T, Bhat S et al. (2012) Are infants born in baby-friendly hospitals being exclusively breastfed until 6 months of age? Eur J Clin Nutr 66, 459-465.

52. Lingam R, Gupta P, Zafar S et al. (2014) Understanding care and feeding practices: building blocks for a sustainable intervention in India and Pakistan. Ann N Y Acad Sci 1308, 204-217.

53. Chhabra R, Subhashini V \& Verma S (2010) Existing infant feeding and weaning practices in an urban setup. Asian J Home Sci 5, 250-254.

54. Narayanappa R, Ranganath $T$ \& Gowda K (2015) Infant feeding practices in rural field practice area of Medical College in Karnataka: a cross-sectional descriptive study. Natl J Community Med 6, 508-512.
55. Collison DK, Kekre P, Verma P et al. (2015) Acceptability and utility of an innovative feeding toolkit to improve maternal and child dietary practices in Bihar, India. Food Nutr Bull 36, 24-32.

56. Roy S, Dasgupta A \& Pal B (2009) Feeding practices of children in an urban slum of Kolkata. Indian J Community Med 34, 362-363.

57. Aggarwal A, Verma S, Faridi M et al. (2008) Complementary feeding - reasons for inappropriateness in timing, quantity and consistency. Indian J Pediatr 75, 49-53.

58. Rasania S \& Sachdev T (2001) Nutritional status and feeding practices of children attending $\mathrm{MCH}$ Centre. Indian J Community Med 26, 145-150.

59. Kuriakose JJR (2010) Nutritional status and feeding practices of infants. Nurs J India 101, 184-186.

60. Yasmin H (2008) Weaning practices of rural women in Chandauli District, Uttar Pradesh, India. Nurture 2, 24-29.

61. Saxena V \& Kumar P (2014) Complementary feeding practices in rural community: a study from block Doiwala district Dehradun. Indian J Basic Appl Med Res 3, 358-363.

62. Padmadas SSS, Hutter I \& Willekens F (2002) Weaning initiation patterns and subsequent linear growth progression among children aged 2-4 years in India. Int J Epidemiol 31, 855-863.

63. Caleyachetty A, Krishnaveni GV, Veena SR et al. (2013) Breastfeeding duration, age of starting solids and high BMI risk and adiposity in Indian children. Matern Child Nutr 9. 199-216.

64. Bhanderi D \& Choudhary S (2011) A community based study of feeding and weaning practices in under five children in semi urban community of Gujarat. Natl J Community Med 2, 277-283.

65. Sreedhara MS \& Banapurmath CR (2014) A study of nutritional status of infants in relation to their complementary feeding practices. Curr Pediatr Res 18, 39-41.

66. Bagul AS \& Supare MS (2012) The infant feeding practices in an urban slum of Nagpur, India. J Clin Diagnostic Res 6, $1525-1527$.

67. Jayant DD, Purushottam AG, Deepak BP et al. (2010) Socio-cultural practices in relation to breastfeeding, weaning and child rearing among Indian mothers and assessment of nutritional status of children under five in rural India. Australas Med J 3, 618-624.

68. Farzani F \& Devi R (2011) A study of feeding practices of infants in Parbhani district. Food Sci Res J 2, 1-3.

69. Meshram II, Kodavanti MR, Chitty GR et al. (2013) Influence of feeding practices and associated factors on the nutritional status of infants in rural areas of Madhya Pradesh State, India. Asia Pac J Public Health 27, NP1345-NP1361.

70. Sanjeev D \& Anuradha D (2012) Women literacy and infant feeding practices in rural integrated child development scheme (ICDS) block of Delhi. Natl J Community Med 3, 385-390.

71. Verma R \& Gupta P (2015) Impact of breast feeding and weaning practices associated with morbidity in rural area of Ghaziabad, Uttar Pradesh, India: a community based longitudinal study. Natl J Community Med 6, 618-621.

72. Damayanthi M, Jayanth Kumar K \& Sridevi (2013) Breast feeding practices in rural field practice area of RRMCH, Bangalore. Med Inn 2, issue 1, 5-8.

73. Pathi S, Das BC, Rasania SK et al. (2003) Feeding practices versus nutritional status of infants in a rural ICDS block of Orissa. Health Popul Perspect Issues 26, 116-120.

74. Shroff MR, Griffiths PL, Suchindran C et al. (2011) Does maternal autonomy influence feeding practices and infant growth in rural India? Soc Sci Med 73, 447-455. 
75. Tyagi R \& Bhan C (2009) Knowledge of rural mothers regarding recommended child rearing practices. Ann Agric Biol Res 14, 169-173.

76. Goswami M, Dash B \& Dash NC (2012) Maternal care and childrearing practices: a micro level study among the Bhumija tribe of Northern Orissa, India. South Asian Anthropol 12, 51-59.

77. Saxena V \& Kumari R (2014) Infant and young child feeding - knowledge and practices of ASHA workers of Doiwala block, Dehradun district. Indian J Community Health 26, 68-75.

78. Padhy K \& Choudhury RKR (2004) Practices and attitude of mothers in initiation of infant feeding. South Asian Anthropol 4, 157-160.

79. Mayuri M, Garg V, Mukherji C et al. (2012) Bovine milk usage and feeding practices for infants in India. Indian J Public Health 56, 75-81.

80. Rao S, Swathi P, Unnikrishnan B et al. (2011) Study of complementary feeding practices among mothers of children aged six months to two years - a study from coastal south India. Australas Med J 4, 252-257.

81. Subbiah N \& Jeganathan A (2012) Socio-cultural beliefs influencing breastfeeding practices among primi postnatal mothers residing in urban slum area of Delhi. Health Popul Perspect Issues 35, 61-73.

82. Sharma M \& Sharma S (2003) Infant feeding practices in rural women of Kangra district of HP. Himachal J Agric Res 29, 79-83.

83. Sinha A \& Pandey H (2000) Weaning practices of 'Ho' mothers in Bihar. Indian J Nutr Diet 37, 338-340.

84. Sinhababu A, Mukhopadhyay DK, Panja TK et al. (2010) Infant- and young child-feeding practices in Bankura district, West Bengal, India. J Health Popul Nutr 28, 294-299.

85. Yousafzai AK, Pagedar S, Wirz S et al. (2003) Beliefs about feeding practices and nutrition for children with disabilities among families in Dharavi, Mumbai. Int J Rehabil Res 26, 33-41.

86. Dahiya S \& Sehgal S (2002) Infant feeding practices of working and non-working urban mothers. Indian J Nutr Diet 39, 367-372.

87. Chhabra P \& Gupta A (2015) Infant and young child feeding practices and its determinants in an urbanized village of Delhi. Int J Med Public Health 5, 228-231.

88. Veena SR, Krishnaveni G V, Srinivasan K et al. (2010) Infant feeding practice and childhood cognitive performance in South India. Arch Dis Child 95, 347-354.

89. Erdman J (1981) Bioavailability of trace minerals from cereals and legumes. Cereal Chem 58, 21-26.

90. Shi L \& Zhang J (2011) Recent evidence of the effectiveness of educational interventions for improving complementary feeding practices in developing countries. J Trop Pediatr 57, 91-98.

91. Dibley MJJ, Roy SKK, Senarath U et al. (2010) Across-country comparisons of selected infant and young child feeding indicators and associated factors in four South Asian countries. Food Nutr Bull 31, 366-375.

92. Ministry of Women and Child Development (2016) Draft National Policy for Women 2016: Articulating a Vision for Empowerment of Women. New Delhi: Government of India.

93. Dewey KG \& Brown KH (2003) Update on technical issues concerning complementary feeding of young children in developing countries and implications for intervention programs. Food Nutr Bull 24, 5-28.

94. Rogers P (2014) Theory of Change. Methodological Briefs Impact Evaluation no. 2. Florence: UNICEF Office of Research - Innocenti.

95. Bhandari N, Mazumder S, Bahl R et al. (2004) An educational intervention to promote appropriate complementary feeding practices and physical growth in infants and young children in rural Haryana, India. J Nutr 134, 2342-2348.

96. Vazir S, Engle P, Balakrishna N et al. (2013) Clusterrandomized trial on complementary and responsive feeding education to caregivers found improved dietary intake, growth and development among rural Indian toddlers. Matern Child Nutr 9, 99-117.

97. Bhutta ZA, Ahmed T, Black RE et al. (2008) What works? Interventions for maternal and child undernutrition and survival. Lancet 371, 417-440.

98. Bahuguna R, Younis Khan S \& Jain A (2013) Influence of feeding practices on dental caries. A case-control study. Eur J Paediatr Dent 14, 55-58.

99. Dakshayani B \& Gangadhar MR (2008) Breast feeding practices among the Hakkipikkis: a tribal population of Mysore District, Karnataka. Ethno-Med 2, 127-129.

100. Fall CHHD, Borja JBB, Osmond C et al. (2010) Infantfeeding patterns and cardiovascular risk factors in young adulthood: data from five cohorts in low- and middleincome countries. Int J Epidemiol 40, 47-62.

101. Holambe VMV \& Thakur NAN (2014) Factors affecting late introduction of complementary food: a Kaplan Meier analysis. Natl J Community Med 5, 140-143.

102. Jindal A (2009) Weaning practices among mothers in selected hospitals in Mangalore. Nurs J India 42, 351356.

103. Kumar D, Goel NKK, Mittal PCC et al. (2006) Influence of infant-feeding practices on nutritional status of under-five children. Indian J Pediatr 73, 417-421.

104. Passi GR \& Shad R (2004) Breastfeeding, weaning practices and nutritional status of infants of tea garden workers of Assam. Indian Pediatr 41, 1277-1279.

105. Shahrawat R \& Joon V (2013) Role of inter personal communication in infant and young child feeding practices in an urban slum: an overview based on case studies. Indian J Pediatr 80, 1041-1046.

106. Singh A \& Vaidya M (2015) Traditional weaning foods and practices in Jabalpur district with reference to prevalence of malnutrition. Asian J Dairy Food Res 34, 32. 